\title{
19 Automatisierter und vernetzter Verkehr in der soziotechnischen Transformation
}

Jens S. Dangschat

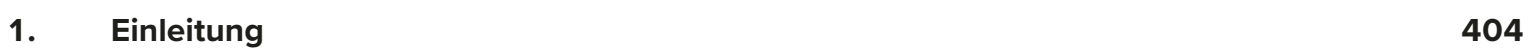

2. Wandel der (modernen) Gesellschaft $\quad 406$

2.1 Der Übergang von der Ersten zur Zweiten Moderne 407

2.2 Bedeutung der (Auto-)Mobilität in modernen Gesellschaften 410

3. Der techniksoziologische Blickwinkel auf technologische und gesellschaftliche Transformationen

3.1 Ansätze der Technikanwendung (Technikdeterminismus) 414

3.2 Ansätze der Technikherstellung 417

$\begin{array}{lll}3.3 & \text { Integrierende Ansätze } & 420\end{array}$

4. Der Ansatz von Geels - und warum er zu kurz greift $\quad 424$

5. "Mobility transfers“ und „policy mobilities“: warum der Entstehungskontext
und die Erzeugung von Bildern für eine kritische Analyse so wichtig sind

6. Digitalisierung und Automatisierung als Treiber des soziotechnischen Wandels? $\quad 430$

$\begin{array}{ll}\text { Literatur } & 433\end{array}$

Jens S. Dangschat

TU Wien, Forschungsbereich Soziologie (ISRA)

jens.dangschat@tuwien.ac.at

(C) Der/die Autor(en) 2021

M. Mitteregger et al. (Hrsg.), AVENUE21. Politische und

planerische Aspekte der automatisierten Mobilität,

https://doi.org/10.1007/978-3-662-63354-0_19 
"[...] we live in a world that not just is changing, it is metamorphosing. Metamorphosis implies a much more radical transformation in which the old certainties of modern society are falling away and something quite new is emerging" (Beck 2016: 3).

\section{EINLEITUNG}

Die Entwicklung des automatisierten und vernetzten Verkehrs (avV) wird - neben Fragen der Ethik, des Rechts, der Sicherheit, der Finanzierung, des Umweltschutzes und der Klimaschonung - vor allem als eine technologische Herausforderung im Rahmen einer breiten und vielfältigen Digitalisierung diskutiert. Gesellschaftliche Auswirkungen oder gar die Einbettung der mit den Entwicklungen des avV verbundenen Techniken in die Gesellschaft spielen demgegenüber in der gegenwärtigen wissenschaftlichen Diskussion zum avV noch eine stark untergeordnete Rolle oder werden bisweilen als hoch unsicher dargestellt.

Dieser Mangel an sozialwissenschaftlichen Forschungen zum avV ist umso verwunderlicher, als die aktuellen gesellschaftlichen Veränderungen sehr stark mit Prozessen der Globalisierung, der Kosmopolitisierung, der Beschleunigung (vgl. Rosa 2005) sowie der Digitalisierung verbunden sind und angenommen werden kann, dass jene Prozesse den Einsatz automatisierter Fahrzeuge stark beeinflussen werden. Die Zukunft vorherzusagen, ist keine traditionelle Grundkompetenz der Soziologie, allerdings sollten anerkannte sozialwissenschaftliche Gegenwartsdiagnosen nicht ausgeblendet und im Bezug zum avV reflektiert werden. Spätestens seit Becks Veröffentlichung über die „Risikogesellschaft“ (Beck 1987) ist innerhalb der Sozialwissenschaften die Rede von der „Enttraditionalisierung der industriegesellschaftlichen Lebensformen“, der „Entstandardisierung der Erwerbsarbeit“ sowie der Individualisierung von Lebenslagen und Biografiemustern innerhalb der „reflexiven Moderne“ (vgl. auch das Konzept der "Liquid Modernity“ von Baumann 2000). Weyer geht in seiner Theorie der „Echtzeitgesellschaft" davon aus, dass in der

"[...] künftigen Gesellschaft [...] tradierte Konzepte nicht mehr greifen, weil
die Grenzen der Planung und Handlung, von Autonomie und Kontrolle,
aber auch von Steuerung und Selbststeuerung zunehmend verschwim-
men" (Weyer 2019: 11).

Kesselring hat aus diesen Überlegungen - mit vielen Querverweisen auf Urrys „mobilities turn“ (vgl. Urry 2000, 2007, 2009; Sheller/Urry 2006, 2016) - das Konzept der „reflexiven Mobilitäten“ entwickelt (vgl. Bonß/Kesselring 2001; Kesselring 2008, 2020). Danach müsse Mobilität u. a. „[...] als ein inkonsistentes, widersprüchliches und ambivalentes Prinzip der Moderne verstanden werden" (Kesselring 2020: 162), was in deutlichem Widerspruch zu den Vorstellungen der traditionellen Verkehrsplanung und -steuerung sowie den klassischen Technik- und Ingenieurswissenschaften steht, in denen Abweichungen von der Linearität rationaler Logik als ReboundEffekt interpretiert werden (vgl. Santarius 2012). Mit dem Ansatz der „Zweiten Moderne“ resp. „reflexiven Moderne“ werden Aspekte wie Unsicherheit, Ambivalenz und Pluralität berücksichtigt, was für das menschliche Handeln neben der Rationalität relevant ist. 
Ein wesentlicher Strang sozialwissenschaftlicher Theoriebildung und Forschung hat sich mit der Parallelität von technologischen, technischen und gesellschaftlichen Prozessen auseinandergesetzt. Saint-Simon (1814) führt beispielsweise die Überwindung der feudalen Gesellschaftsordnung auf das erstarkte Bürgertum zurück, das „sein Selbstbewusstsein auf ökonomische, technische und wissenschaftliche Erfolge gründet" (Häußling 2014: 13). Die soziologische Fragestellung sei daher, „wie soziales Zusammenleben, gesellschaftliche Normen und Strukturen sowie sozialer Wandel durch die Einbindung von Technik funktionieren“ (ebd.: 13). Auch Marx (1867/1969) leitet die gesellschaftlichen Umbrüche wie die Neuformierung der Klassenstrukturen oder den soziokulturellen Wandel („Bewusstsein“) und neue Handlungsmuster u. a. von der Technisierung der Arbeitswelt ab. Schumpeter (1942) sieht als Treiber des Konjunkturzyklus das Wechselspiel zwischen unterschiedlich innovativen Unternehmerlnnen an, in dessen Kontext der technisch-wirtschaftliche Fortschritt für den Erfolg von Produkten, Unternehmen und Volkswirtschaften zentral ist. Auf Schumpeters Begriff des „kreativen Zerstörers“ aufbauend, hat Christensen (1997) den Begriff der "disruptiven Technologie“ eingeführt.

Digitalisierung und die darin eingebettete Automatisierung und Vernetzung des Verkehrs werden meist von international tätigen großen Beratungsunternehmen als ein „disruptiver technologischer Wandel“ eingeordnet (vgl. auch Jonuschat et al. 2016). Wenn aber die Veränderungen, die mit den teils noch zu entwickelnden Technologien und insbesondere den vielfältigen Anwendungen einhergehen, so fundamental sind, dann ist es unerlässlich, diese Entwicklungen in ihrer gesellschaftlichen Bedeutung zu analysieren. Aber gerade in den Perioden stärkerer gesellschaftlicher Veränderungen liefern sozialwissenschaftliche Analysen unterschiedliche Deutungen. Um politisch-planerische, aber auch unternehmerische Entscheidungen zu treffen, die am Gemeinwohl, an unternehmerischer Stabilität und an einer nachhaltigen Entwicklung orientiert sind, ist es notwendig, den Aufbau, die Differenzierung und die Dynamik der aktuellen Gesellschaften zu erkennen.'

Das setzt im ersten Schritt voraus, sich über das Ausmaß und die Dynamik des gesellschaftlichen Wandels zu verständigen. Das impliziert, die wesentlichen Treiber gesellschaftlicher Veränderungen zu berücksichtigen und deren Einflüsse zu bewerten (siehe Abschnitt 2). Wie sich die Soziologie mit dem Einfluss technologischer und technischer Entwicklung befasst hat, wird in Abschnitt 3 gezeigt. Der in der Wissenschaft breit diskutierte Ansatz von Frank W. Geels (2004), die Makro-Meso-Mikro-Verknüpfung soziotechnischer Innovationen zu analysieren, wird in Abschnitt 4 kritisch eingeordnet. Im Anschluss daran wird argumentiert, wie notwendig es ist, die politische Erzeugung von Vorstellungen über den technischen Fortschritt im Allgemeinen und die Automatisierung und Vernetzung des Verkehrs im Besonderen kritisch zu analysieren (siehe Abschnitt 5). Abschließend wird ein Ausblick auf die „digitale Moderne“ (vgl. Canzler/Knie 2016) gerichtet und betrachtet, welche Rolle der avV bei deren Neufiguration spielen wird (siehe Abschnitt 6).

1 Politik und öffentliche Verwaltungen stützen sich bei ihrer Analyse der Gesellschaft vor allem auf die amtliche Statistik. Diese liefert jedoch hinsichtlich der Ausdifferenzierung der Gesellschaft eine völlig unzureichende Datenlage, weil die wichtige soziokulturelle Dimension völlig fehlt und die sozioökonomische nur oberflächlich abgebildet wird. Dieser „Blindflug“ ist nicht nur aufgrund mangelnder Differenziertheit problematisch, sondern er trägt auch dazu bei, dass weiterhin in den veralteten Kategorien der „Ersten Moderne“ gedacht und gehandelt wird (vgl. Dangschat 2015). Zudem ist eine Wissenschaft, die auf diesen Daten und ihrer Verräumlichung in administrativen Einheiten angewiesen ist, deutlich benachteiligt, was sich letztlich negativ auf die sozialwissenschaftliche Expertise in der Politik-, Planungs- und Unternehmensberatung auswirkt. 


\title{
2. WANDEL DER (MODERNEN) GESELLSCHAFT
}

\author{
„Um die gesellschaftliche Sprengkraft der digitalen Transformation zu er- \\ fassen, ist es sinnvoll, in größeren gesellschaftlichen Zusammenhängen zu \\ denken" (Weyer 2019: 10).
}

Es besteht innerhalb der Sozialwissenschaften kein Zweifel darüber, dass sich zumindest moderne Gesellschaften und jene der wirtschaftlichen Schwellenländer aktuell in einer Phase eines raschen Wandels befinden: hinsichtlich der Institutionen, Organisationen, Strukturen, Einstellungen und Verhaltensweisen (vgl. Sennett 1998). In diesem Kontext verliere der Nationalstaat als wirtschaftslenkende und sozial ausgleichende Ordnungsmacht an Bedeutung ${ }^{2}$, während sich Unternehmen mit zunehmenden Interdependenzen und Abhängigkeiten im globalen Maßstab vernetzen und eigene Dynamiken entwickeln.

Die sozialen Strukturen verändern sich auseinanderstrebend in den sozioökonomischen, soziodemografischen, soziokulturellen und sozialräumlichen Dimensionen. Die Gesellschaften befinden sich in einem Wertewandel mit zunehmend gegensätzlichen Positionen und partikularen Interessen, wobei sich die Lebensstile - vor dem Hintergrund der sich verschärfenden Gegensätze materieller und zeitlicher Ressourcen - global im Sinne bildungsnaher urbaner Mittelschichten angleichen (vgl. Dangschat 2020). Gerade der Wertewandel sorge für "multiple Modernitäten“ (vgl. Eisenstadt 2006).

Als Ursachen dieser Entwicklungen wurden die Begriffe der Globalisierung (vgl. Sassen 2001, Wallerstein 2004), Beschleunigung (vgl. Rosa 2005), Individualisierung (vgl. Beck 1995), Flexibilität (vgl. Sennett 1998), Digitalisierung (vgl. Elliott 2018), Vernetzung (vgl. Castells 2001) und Mobilität (vgl. Urry 2000) in der Fachliteratur eingeführt und mit zeitlicher Verzögerung über den medialen Diskurs zum Bestandteil einer allgemeinen gesellschaftlichen Interpretation.

In der deutschsprachigen Forschung zur sozialen Ungleichheit gibt es drei grundlegende Strömungen darüber, wie die gesellschaftlichen Prozesse zumindest in den postindustriellen Ländern hinsichtlich der Formen sozialer Ent- und Restrukturierung einzustufen sind (vgl. Dangschat 2020):

- als eine Verfestigung und Wiederbelebung von bestehenden Klassenstrukturen unter gewandelten Erscheinungsformen; die neoliberale globale Wirtschaft zeigt nicht nur zwischen dem Globalen Norden und dem Globalen Süden, sondern auch vor allem innerhalb der Nationalstaaten neue Klassenverhältnisse (vgl. Dangschat 1998);

- als „Entbettung“ aus traditionellen Bindungen (Herkunft, Bildung, soziale Lage, GenderRollenverständnis etc.) bei gleichzeitiger „Rückbettung“ in neue Formen soziokultureller Unterschiede (soziale Milieus, Lebensstile; vgl. Vester et al. 2001) oder

2 Zu Beginn der Corona-Pandemie gab es in den meisten europäischen Demokratien jedoch eine deutliche Gegenbewegung mit einer starken Zunahme des Vertrauens in den Nationalstaat und die Regierungsparteien, obwohl (oder weil) sie tief in die Grundrechte eingegriffen hatten. Die Phase der Lockerung zeigt jedoch, dass diese „Gegenbewegung“ von kurzer Dauer war und nicht nur partikulare Interessen wieder in den Vordergrund treten, sondern sich auch der Raum für unterschiedliche Verschwörungsthesen und Falschmeldungen weiter öffnet. 
- auch als „Entbettung“ aus den traditionellen Bindungen, aber ohne Möglichkeit, dauerhaft auf neue kollektive Orientierungen zurückgreifen zu können, und stattdessen darauf angewiesen zu sein, sich selbst permanent neu zu verorten und zu vergewissern (Übergang von der Ersten zur Zweiten Moderne; vgl. Beck et al. 2001).

Diese drei idealtypischen Ansätze stehen als „Schulen“ einander gegenüber und unterscheiden sich, indem sie aus kapitalismuskritischer Sicht die veränderten Erscheinungsformen (von der Erosion des Sozialstaates bis hin zu individuellen Konsumstilen) hervorheben, die neuen Ebenen der Vergemeinschaftung und Vergesellschaften betonen oder aber die Gesellschaften als in einem fundamentalen Übergang befindlich betrachten, d. h., sie unterscheiden sich vor allem in der Einschätzung, wie tiefgreifend die gesellschaftlichen Veränderungen sind resp. künftig sein werden.

In diesem Beitrag soll der dritte Ansatz verfolgt werden, weil dort explizit angenommen wird, dass die technologischen und technischen Transformationen, die im Rahmen der Digitalisierung absehbar ablaufen werden, einen massiven Einfluss auf Gesellschaften haben werden. Zudem wird innerhalb der These des Übergangs in die Zweite Moderne - auch „reflexive Moderne“ genannt (vgl. Beck et al. 1996, Beck et al. 2001) - der Begriff der Mobilität in einen sehr weitreichenden, den Verlauf der Moderne bestimmenden Kontext gestellt, der den Blick auf die gesellschaftliche Einordnung einer neuen Mobilitätstechnologie wie der des avV noch einmal schärfen kann (vgl. Bonß/Kesselring 2001, Kesselring 2020).

In dieses erweiterte Verständnis fließen zum einen die Überlegungen von Urry und Sheller zum „mobilities turn“ ein, welche die Soziologie wesentlich beeinflusst haben (vgl. Urry 2000, 2007, 2009; Scheller/Urry 2006, 2016; Sheller 2011). Zum anderen wird der Diskurs des „space of flows“, d. h. der Neuorganisation räumlicher Arrangements vor dem Hintergrund technologisch bedingter „Echtzeiten“ (vgl. Castells 1989) ebenso aufgenommen wie der der „(socio-)spheres“, d. h. der Auflösung nationalstaatlicher Bedeutung (vgl. Albrow 1998), der „scapes“, d. h. der „enträumlichten“ Landschaften (vgl. Appadurai 1996, Urry 2003), der „fixities“ und „motion“ (vgl. Brenner 1998), sowie die Einschätzung moderner Gesellschaften als Netzwerkgesellschaften (vgl. Castells 2001).

\subsection{DER ÜBERGANG VON DER ERSTEN ZUR ZWEITEN MODERNE}

Die Entwicklung der Moderne steht also in einem engen Zusammenhang mit der technologischen Entwicklung und der Mobilität. Im Verlauf der Moderne haben sich - wenn auch in Schüben -

- der Aufbau der Gesellschaften (Ausdifferenzierung der Schichten durch die Arbeitsteilung und einer Dominanz der - urbanen - Mittelschichten),

- die industrielle Produktion (Fordismus auf Basis der Fließbandproduktion, Taylorismus),

- die Technologien (als Abfolge der Dominanz von Schlüsseltechnologien wie in den Kondratieff'schen Zyklen dargestellt) sowie

- politische Prozesse (Demokratisierung, Formen der Sozialstaatlichkeit im Zuge des Keynesianismus), die Bürokratie und das Bildungssystem (verpflichtendes Schulsystem für alle)

kontinuierlich verändert. 
In den 1970er Jahren entstand in vielen Wissenschaften und der Kunst eine zunehmende Skepsis gegenüber der Annahme der Linearität und Zielgerichtetheit der Modernisierungsprozesse. Das „Ende der Moderne“ wurde ausgerufen und durch unterschiedliche „Post-Modelle“ ersetzt: Postmoderne, Postfordismus, postindustriell, Postkolonialismus, Poststrukturalismus, Postwachstum etc. Man spricht auch von „Kehrtwenden“ wie dem „cultural turn“, „communicative turn“ oder „mobility turn“. Während die „Post“-Begriffe lediglich darauf hinweisen, dass etwas zu Ende und in der weiteren Diskussion umstritten ist, wie die aktuellen und denkbaren Zukünfte zu verstehen seien, gehen die „Turn“-Ansätze von einem Umdenken aus, meist als Abkehr von den dominant linear-logischen Vorstellungen. Beispielsweise ist Lash (1999) der Auffassung, dass die „neue Moderne“ mit einer veränderten Form der Rationalität einhergehe, was insbesondere die positivistische Position herausfordere.

Abweichend von diesen Auflösungen und auch Neupositionierungen beharrt der disziplinäre Mainstream der Soziologie auf der These einer fortschreitenden Modernisierung. Gegen diesen Mainstream hat der Soziologe Ulrich Beck wiederholt und beharrlich argumentiert: Er votiert zum einen für eine Betrachtung der sozialen Ungleichheit "jenseits von Klasse und Stand“ (Beck 1983) und sieht die moderne Gesellschaft als eine "Risikogesellschaft" (Beck 1987), für deren Überwindung ein „Weg in eine andere Moderne“ (Untertitel) notwendig sei. Erst später bezeichnet er diesen Ansatz als „Zweite Moderne“ resp. „reflexive Moderne“ (Beck et al. 1996, 2001).

Gerade die technologischen Entwicklungen, die kapitalistische Logik und das Ausblenden der zunehmend problematischen klimatischen und ökologischen Prozesse führen nach seiner Einschätzung aufgrund der Logik der (traditionellen) Moderne zunehmend zu industriellen Katastrophen, die mit den Möglichkeiten, aber auch den eingefahrenen Strategien der traditionellen Moderne nicht nur nicht mehr zu verhindern seien, sondern die sich zunehmend verschärfen ${ }^{3}$. Es bedürfe hingegen einer neuen Nachdenklichkeit, neuer Formen des politischen Aushandelns (vgl. Hajer/Wagenaar 2003) und des Vertrauens in Prozesse statt in Strukturen und Institutionen.

Beck kritisiert die FachkollegInnen zudem aufgrund des Festhaltens am Denken in Kategorien nationalstaatlich verfasster Gesellschaften. Die globale Vernetzung von Produktions-, Handelsund vor allem Finanzkapitalströmen unterlaufe die Gestaltungs- und Steuerungsmacht der traditionellen Industriestaaten zugunsten transnational agierender Konzerne (vgl. Beck 1997). Die Folge seien eine Schwächung des Sozialstaates, gesellschaftliche Desintegrationsprozesse, die Erosion vieler vertrauter Sozialbeziehungen und verstärkte „Freisetzungen“ aus gewohnten sozialen Kontexten auf der individuellen Ebene, welche Beck mit seiner „Individualisierungsthese" fasst (vgl. Beck 1995).

Auch die Wissenschaften sieht Beck im Umbruch: Seiner Auffassung nach werden sie in der Zweiten Moderne nicht nur für Problemlösungen herangezogen, sondern sind zugleich auch Problemverursacher, denn wissenschaftliche Analysen hätten u. a. aufgrund einer unüberschaubaren Flut an unzusammenhängenden Detailergebnissen im Zuge der praktischen Umsetzung auch fragwürdige Seiten gezeigt. Daraus entstehe zum einen eine Unsicherheit durch teils widersprüchliche Ergebnisse, zum anderen könnten sich Rezipienten aller gesellschaftlicher Bereiche „ihre Wahrheiten“ aus einem inkonsistenten wissenschaftlichen Angebot heraussuchen - die Diskussion um die Dynamik und die Art des Ausstieges aus dem coronabedingten Shut-down sind ein gutes Beispiel hierfür.

3 Beck hatte seinerzeit insbesondere die Explosion des Chemiewerkes im indischen Bhopal und ihre verheerenden Folgen im Jahr 1984 vor Augen. Seine Publikation erschien zudem im Mai 1987, nur wenige Tage nach der Explosion des Kernkraftwerkes in Tschernobyl. 
Die gemeinsame Arbeit mit Lash und Giddens an der „reflexiven Moderne“ (vgl. Beck et al. 1996) und die parallelen Arbeiten von Sennett (1998) führten über mehrere Jahre zu einer sehr fruchtbaren Zusammenarbeit der vier Wissenschaftler an der London School of Economics (LSE). Mit seinen Kollegen formulierte Beck dabei die sehr weitreichende Hoffnung, dass es den Menschen gelingen werde, ihre Zukunft dadurch „vernünftig“ zu gestalten, und dass auf der Grundlage einer Analyse der gegenwärtigen (globalen) Probleme Verbesserungsansätze entwickelt werden.

Kesselring hat in seiner Reflexion der Bedeutung der Mobilität innerhalb der Ersten und der Zweiten Moderne die Unterschiede zwischen beiden wie folgt einander gegenübergestellt (siehe Übersicht 1).

Übersicht 1: Kriterien zur Unterscheidung in die Erste und Zweite Moderne

\begin{tabular}{|c|c|c|}
\hline & Erste Moderne & Zweite Moderne \\
\hline Reaktion auf Ambivalenz & Purifikation & Pluralismus \\
\hline Optimale Lösung & „One-best-Way“-Lösung & $\begin{array}{c}\text { „Multiple-best-Way“- } \\
\text { Lösung }\end{array}$ \\
\hline Ordnungskategorien & Strukturen, Regeln, Beständigkeit & $\begin{array}{c}\text { Netzwerke, „Scapes“, } \\
\text { „Flows“ }\end{array}$ \\
\hline Absicherung & Sicherheit und Gewissheit & Risiko und Ungewissheit \\
\hline Wissenschaftliche Eindeutigkeit & $\begin{array}{l}\text { Vorherseh- und -sagbarkeit, } \\
\text { wachsende Stabilität }\end{array}$ & $\begin{array}{l}\text { Unvorhersehbarkeit, } \\
\text { wachsende Flüchtigkeit }\end{array}$ \\
\hline Struktur und Dynamik & Kontinuität und Entwicklung & Diskontinuität und Wandel \\
\hline Orientierung & zielorientiert & prozessorientiert \\
\hline Scale & nationale Ordnung & $\begin{array}{l}\text { kosmopolitische } \\
\text { Kontingenz }\end{array}$ \\
\hline Verbindungen & dauerhafte, stabile Verbindungen & $\begin{array}{c}\text { Konnektivität als Problem } \\
\text { und Projekt }\end{array}$ \\
\hline Ordnung & $\begin{array}{l}\text { dauerhafte, auf Stabilität ausge- } \\
\text { richtete (nationale) Strukturen und } \\
\text { Ordnung }\end{array}$ & $\begin{array}{l}\text { temporäre, transitive } \\
\text { (transnationale) } \\
\text { Strukturierung und } \\
\text { Unordnung }\end{array}$ \\
\hline Grenzen & $\begin{array}{l}\text { feste Grenzen und } \\
\text { Aufrechterhaltung der Grenzen }\end{array}$ & $\begin{array}{l}\text { flexible Grenzen } \\
\text { und flexibles } \\
\text { Grenzmanagement }\end{array}$ \\
\hline
\end{tabular}

Quelle: eigene Darstellung nach Kesselring (2020: 178)

Aus dieser Gegenüberstellung wird ein Spannungsverhältnis für die Ausrollung des avV deutlich: Die technologische Entwicklung der Fahrzeuge und der Infrastrukturen ist ebenso fest in den Vorstellungen der Ersten Moderne verankert wie politische Strategiepapiere und ingenieurwissenschaftliche Publikationen (klare Strukturen, Rationalität, Gewissheit, Vorhersagbarkeit etc.). Sie treffen aber bereits jetzt und künftig noch eindeutiger auf einen gesellschaftlichen Kontext, der durch die Zweite Moderne beschrieben wird: Diskontinuität, transitive Strukturierung, Ungewissheit und Risiko (siehe Abschnitt 6). 


\subsection{BEDEUTUNG DER (AUTO-)MOBILITÄT IN MODERNEN GESELLSCHAFTEN}

Mobilität wird in diesem Diskurs als ein grundlegendes Prinzip der Moderne angesehen (vgl. Kesselring 2020: 161). Nach Sennett (1977) wird in der Moderne eine uneingeschränkte individuelle Beweglichkeit als ein „Grundrecht“ für alle BürgerInnen aufgefasst. „Freie Fahrt für freie Bürger" war in den 1970er Jahren nicht nur ein Slogan des ADAC, sondern auch eine politische Forderung des größten Vereins in Deutschland. Eine Reihe von Prozessen wie Rationalität, Individualität, Globalisierung, Beschleunigung, Wettbewerb und Freizügigkeit, welche die Modernisierung begleiten und formen, haben begünstigt, dass sich in modernen Gesellschaften die „Automobilität“ herausbilden konnte.

Automobilität ist mehr als nur die Benutzung von Autos, sondern sie ist ein komplexes, sich selbst verstärkendes soziomaterielles System aus technologischen und kulturellen Prozessen, Politiken, Normen und Praktiken. Moderne Gesellschaften sind demnach unabänderlich in die Benutzung fossil angetriebener Autos, deren langlebige Infrastrukturen und suburbane Siedlungsstrukturen sowie in die Politik der Unterstützung traditioneller Industrien sowie in die kulturellen Erwartungen und Erfahrungen mit der Organisation des Alltages in Raum und Zeit eingebunden.

Automobilität besteht nach Urry (2004: 26f.) aus dem Zusammenspiel von sechs Komponenten, welche den „spezifischen Charakter der Dominanz“ ausmachen:

1. das Auto als Fahrzeug, das von den „ikonischen“ Unternehmen des wichtigsten Sektors der Industrialisierung im 20. Jahrhundert hergestellt wird, und

2. welches nach dem Wohnen das wichtigste Konsumgut ist, das den Nutzerlnnen bzw. Besitzerlnnen durch seine symbolische Aufladung einen Status vermittelt.

3. Durch die technischen und sozialen Verbindungen mit den Industrien entsteht ein mächtiger Komplex von Infrastrukturen, Reparatur- und Freizeitbetrieben, welcher über den Städtebau und die Stadtplanung hergestellt wird;

4. Das Auto verkörpert die globale Form einer „quasiprivaten“ Mobilität, welche das berufliche und private Leben gestaltet und dabei andere Formen der Mobilität dominiert und zurückdrängt.

5. Es definiert eine Kultur des guten Lebens und der angemessenen bürgerlichen Mobilität.

6. Durch die verwendeten Materialien, die Energie und den Raum bei der Produktion der Fahrzeuge, die Kosten und den Raum der Straßen, der Infrastrukturen sowie durch die Emissionen und die gesundheitlichen und die sozialen Folgekosten ist das Auto der größte Verbraucher natürlicher Ressourcen.

Ähnlich argumentieren Canzler und Knie (2019):

„[Das] [...] Auto wurde zu einem Teil des Alltags; es strukturierte mehr und mehr auch die Lebensplanungen und schaffte neue, ungeahnte Möglichkeitsräume. Wo arbeiten? Wo wohnen? Mit dem Auto gab es plötzlich viel mehr Optionen. Der Stadtrand und der ländliche Raum wurden als Siedlungsgebiete erschlossen, das Pendeln zum Arbeitsort als Normalfall erfunden. Gesellschaftliche Teilhabe hing nicht mehr vom Wohnort ab, das Auto ermöglichte neue Zugänge auch im privaten Bereich, die Überwindung des Raums wurde sozial erschwinglich" (Canzler/Knie 2019: 14). 
Mit dem automatisierten Fahrzeug, das als smart, sauber, sicher und sozial integrativ dargestellt wird, soll dem aufkommenden negativen Image entgegengearbeitet werden. Per se ist der Ersatz eines konventionellen Fahrzeuges durch ein automatisiertes kein Beitrag zu einer nachhaltigeren Mobilität. Auch wenn die Entwicklung im Zusammenhang mit einer „Antriebswende“ steht, ist zu befürchten, dass die Mobilitätswende durch den avV behindert werden wird (vgl. Manderscheid 2020).

In der Verkehrsforschung der Ersten Moderne dominiert - neben der Sicherheit und Verlässlichkeit - vor allem die Rationalität der kosten- und zeitgünstigen Überwindung von Distanzen. Verkehr wird in Kategorien der "Leistung“ erfasst, der die „Leistungsfähigkeit“ der Infrastruktur gegenübergestellt wird. Werden aufgrund von Prognosen Einschränkungen der Leistungsfähigkeit durch ein erhöhtes Verkehrsaufkommen im Zuge des motorisierten Individual- und des Güterverkehrs befürchtet, sei die Infrastruktur vorausschauend auszubauen. Die Gestaltung des Straßenraumes wurde diesem Prinzip zulasten anderer Nutzungen weitgehend untergeordnet.

Durch eine Verlagerung des zunehmenden Interesses hin zur Mobilitätsforschung wurde mit der Linearität der ingenieur- und technikwissenschaftlich dominierten Verkehrsforschung gebrochen, indem das Schwergewicht auf die handelnden AkteurInnen gerichtet wurde. Bei den psychologisch und soziologisch beeinflussten Ansätzen überwiegt die Anwendungsseite und erklärt zum einen „irrationales“ Handeln, zum anderen die Mobilität unterschiedlicher sozialer Gruppen (vgl. Scheiner 2009; Dangschat 2013, 2017b).

Aus dem sehr unterschiedlichen Mobilitätsverhalten sozialer Gruppen entstehen aufgrund subjektiv motivierter Optimierung Ambivalenzen. Demnach ist Mobilität in der Zweiten Moderne nicht mehr nur rational, sondern vielfältig, nicht mehr linear, sondern eher nichtlinear (vgl. Kesselring 2020: 172-175). Die damit verbundene Pluralität lässt sich jedoch weniger denn je in einen einheitlichen Transformationsprozess (zu einer nachhaltigen Mobilität) überführen. Schneidewind et al. (2018: 11) sprechen in diesem Zusammenhang von einer notwendigen besonderen (transformativen) „literacy“, d. h. einer Fähigkeit, die unterschiedlichen Motivationen und Handlungsweisen zu verstehen und politisch-planerisch zu lenken. Wenn die „reflexive Modernisierung" unerwartet, unbemerkt und ungewollt verläuft, dann trifft die rationale Planung auf ihre Grenzen (vgl. Kesselring 2020: 172).

In ihren Überlegungen zu technologischen Transformationen haben sich Sovacool und Axsen (2018) damit auseinandergesetzt, wie die Elektrifizierung, das Sharing und die Automatisierung das Verkehrssystem und damit auch die Automobilität verändern werden. Sie ordnen dabei die acht Aspekte der Automobilität (,frames“) in ein Vier-Felder-System aus individueller vs. gesellschaftlicher Bedeutung und funktionaler vs. symbolischer Effekte ein (siehe Übersicht 2).

Aus der Übersicht wird deutlich, dass durch den avV die Automobilität eher gestärkt als abgeschwächt werden wird. Sovacool und Axsen (2018: 740) geben auch eine Einschätzung über die Stärke der Evidenz ihrer Einschätzungen ab. Ich schätze sowohl die Umweltverantwortung als auch die Ölabhängigkeit etwas anders als die beiden Autoren ein (siehe die mit " "“" versehenen Frames in der Übersicht 2). Zudem halten sie eine Reihe ihrer Einschätzungen für nicht oder nicht ausreichend gesichert, wie beispielsweise die Nutzung als digitales Büro und als Symbol für Wohlstand und sozialen Status.

Die Eigendynamik technologischer und technischer Entwicklung des avV wird also aus sich heraus kaum einen eigenständigen Beitrag zur Abschwächung des Klimawandels, des Flächenund Ressourcenverbrauchs sowie der gesellschaftlichen Symbolik der Automobilität leisten (vgl. Mitteregger et al. 2020: 148ff). Im Gegenteil: Die Automobilität wird durch die „Neuerfindung" des Pkw als sicher, smart, innovativ und sauber gestärkt werden. Da durch die Automatisierung und Vernetzung die Ziele einer nachhaltigen Verkehrsentwicklung mit hoher Wahr- 
scheinlichkeit unterlaufen werden (vgl. Dangschat 2017a, 2019; Milakis et al. 2017; Dangschat/ Stickler 2020), ist es die Aufgabe der Politik und planenden Verwaltung, frühzeitig entsprechende Rahmenbedingungen für die Entwicklung und die Ausrollung automatisierter und vernetzter Fahrzeuge sowie der damit verbundenen Infrastruktur zu setzen.

Übersicht 2: Auswirkungen automatisierter und vernetzter Fahrzeuge auf Aspekte der Automobilität

\begin{tabular}{|c|c|c|}
\hline Frame & Type & $\begin{array}{l}\text { Autonomous Vehicles } \\
\text { (SAE } 4 \text { and 5) }\end{array}$ \\
\hline cocooning and fortressing & private-functional & strengthened \\
\hline mobile digital offices & private-functional & strengthened \\
\hline expression of gender identity & private-symbolic & maintained \\
\hline expression of class and wealth & private-symbolic & $\begin{array}{l}\text { maintained, perhaps } \\
\text { strengthened }\end{array}$ \\
\hline environmental stewardship & societal-functional & $\begin{array}{l}\text { weakened, as it leads to } \\
\text { increased energy use* }\end{array}$ \\
\hline suburbanizing & societal-functional & $\begin{array}{l}\text { strengthened, as it leads to } \\
\text { longer commute distance }\end{array}$ \\
\hline oil independence & societal-symbolic & independent* \\
\hline innovativeness & societal-symbolic & strengthened \\
\hline
\end{tabular}

* vom Original abweichende Einschätzung des Autors Quelle: eigene Darstellung nach Sovacool/Axsen (2018: 740f.)

\title{
3. DER TECHNIKSOZIOLOGISCHE BLICKWINKEL AUF TECHNOLOGISCHE UND GESELLSCHAFTLICHE TRANSFORMATIONEN ${ }^{5}$
}

\begin{abstract}
„Technik [besitzt] nicht per se eine Bedeutung [...], sondern [...] die Bedeutung [wird] mehr oder weniger kontrovers zwischen den Anspruchsgruppen ausgehandelt“ (Häußling 2014: 240).
\end{abstract}

Die Einführung des automatisierten und vernetzten Verkehrs gilt als eine grundlegende, teils disruptive technologische Entwicklung, welche das Verkehrssystem, die Mobilität, die Raumnutzung und weitere gesellschaftliche Entwicklungen stark beeinflussen wird (vgl. Braun et al. 2019). Gerade die Techniksoziologie hat sich mit dem Wechselverhältnis aus technologischen/technischen

$4 \quad$ Mit den Standards der SAE International werden Stufen der Automatisierung beschrieben; danach werden mit dem SAE4-Standard hoch- und mit dem SAE5-Standard vollautomatische Fahrzeuge bezeichnet

$5 \quad$ Einen umfangreichen Überblick zu den unterschiedlichen Ansätzen und Fragestellungen geben Weyer (2008) und Häußling (2014: 129-354). 
und sozialen Entwicklungen auseinandergesetzt. In dieser speziellen Soziologie werden insbesondere im internationalen Diskurs die Begriffe „Technik“ und „Technologie“ häufig synonym verwendet. Innerhalb dieses Beitrages werden mit dem Begriff „Technik“ Verfahren beschrieben, mit denen die Erkenntnisse der Naturwissenschaften praktisch nutzbar gemacht werden ${ }^{6}$. „Technologie" ist hingegen die Lehre von der Technik und befasst sich auf der Basis naturwissenschaftlichtechnischer und ingenieurwissenschaftlicher Erkenntnisse mit der Art und Weise, wie Roh- und Werkstoffe in Produktionsverfahren und fertige Produkte umgewandelt werden.

Rammert (2007: 18) unterscheidet drei grundlegende Perspektiven der Techniksoziologie, die sich in kritischer Abgrenzung innerhalb der letzten Jahrzehnte entwickelt haben:

- Technikdeterminismus: Danach ist Technik die entscheidende Größe einer breit verstandenen gesellschaftlichen Entwicklung (gesellschaftliche Folgen der Technik bzw. Technikentwicklung; siehe Abschnitt 3.1).

- Sozialkonstruktivismus: Demzufolge bestimmen gesellschaftliche Konstruktionen sowie institutionelle, wirtschaftliche und kulturelle Institutionalisierungen die Genese und die Gestalt von Techniken (siehe Abschnitt 3.2.2).

- Interaktionistische Perspektive (Pragmatismus) resp. assoziationistische Perspektive (Akteur-Netzwerk-Theorie; siehe Abschnitt 3.3): Technik und Gesellschaft bilden eine Einheit wechselseitiger Durchdringung.

Der Ökonom Möhrle (2018) definiert als soziologisches Verständnis von Technologie die

„systematische Zusammenfassung und Integration einzelner Techniken zu einer auf spezifische Ziele und Zwecke gerichteten Verfahrensweise, einschließlich sozialer Technologien, z. B. einem Verfahren der Konfliktregelung. Moderne Technologien bestimmen und gestalten die sozialen Beziehungen und den sozialen Wandel in hohem Maße; sie können daher nicht isoliert von der Gesellschaft betrachtet werden und müssen auf ihre Sozialverträglichkeit hin bewertet werden".

Diese Definition zeigt einen deutlichen technikdeterministischen Anwendungsbezug, wie er in den neoklassischen Wirtschaftswissenschaften vorherrscht. Aktuelle Ansätze trennen allenfalls analytisch zwischen beiden Bereichen, gehen aber hinsichtlich ihrer Dynamik von einer wechselseitigen Durchdringung und Beeinflussung aus (siehe Abschnitt 3.3). Bezogen auf autonome und vernetzte Fahrzeuge (bis einschließlich SAE-Stufe 4) bedeutet das, auf der Individualebene der Anwendung die Mensch-Maschine-Schnittstellen zu analysieren und zu gestalten. Für die Herstellung der Technologien auf der Makroebene sind jedoch die technologischen, ökonomischen, wissenschaftlichen und gouvernmentalen Narrationen ebenso bedeutsam wie die staatlichen Regulationssysteme und die Marketingstrategien (siehe Abschnitt 5 und 6).

Um eine fundierte Analyse des Wechsel- und Durchdringungsverhältnisses von technologischen und gesellschaftlichen Entwicklungen leisten zu können, ist es ratsam, sich an techniksoziologischen Theorien und Methoden zu orientieren (siehe Abschnitt 3.2 und 3.3). Häußling (2014: 16) definiert den Gegenstand der Techniksoziologie wie folgt:

6 Ein weiteres, vor allem umgangssprachliches Verständnis von Technik beschreibt bestimmte festgelegte Vorgehensweisen, Methoden und Fertigkeiten (Atemtechnik, Redetechnik, Massagetechnik, Maltechnik etc.), die hier jedoch nicht berücksichtigt werden. 
„Die Techniksoziologie befasst sich mit den Wechselbezügen zwischen Realtechniken, Prozesstechniken und Technologien einerseits und Gesellschaft und Sozialem andererseits. Dabei kann sowohl der Anwendungskontext als auch der Herstellungskontext von Technik im Fokus stehen. Bei dem erstgenannten Kontext geht es um Aneignungsprozesse von Technik [...]. Bei dem zweiten Kontext stehen Aushandlungsprozesse bei der Technologieentwicklung im Vordergrund."

Wenn oben konstatiert wurde, dass sozialwissenschaftliche gegenüber technologischen Betrachtungen des avV deutlich seltener vorgenommen werden, so wird nach dieser Definition deutlich, dass sich fast alle sozialwissenschaftlichen Betrachtungen des avV bislang vor allem auf die (potentiellen) Anwendungs- bzw. Aneignungsprozesse beziehen (zu Ausnahmen vgl. Dangschat/Stickler 2020; Stickler 2020b resp. Manderscheid 2012, 2014, 2020, die jedoch vor allem die Auswirkungen des technologischen Wandels auf die Automobilität betrachtet).

In der folgenden Beschreibung unterschiedlicher Ansätze wird im ersten Schritt in Ansätze der Technikanwendung (siehe Abschnitt 3.1) und der Technikherstellung (siehe Abschnitt 3.2) unterschieden. Im Abschnitt 3.3 wird der aktuell dominierende Ansatz der „Science and Technology Studies" und hier insbesondere die Theorie großer technischer Systeme und der Ansatz der Akteur-Netzwerk-Studien beschrieben.

\subsection{ANSÄTZE DER TECHNIKANWENDUNG (TECHNIKDETERMINISMUS)}

Mit den Ansätzen der Technikanwendung wird eine Perspektive eingenommen, wonach sich Technologien resp. technische Systeme auf die Gesellschaft auswirken (z. B. auf Arbeitsabläufe, Gesundheit, Mobilität, Kommunikation, aber auch regionale und soziale Ungleichheiten). Wenn die Position vertreten wird, dass ein technologischer den gesellschaftlichen Wandel wesentlich beeinflusst, spricht man von „technikdeterministischen Positionen“. Prominente Vertreter sind Schumpeter und Ogburn; auf Letzteren geht der Ansatz der Technikfolgenabschätzung zurück (vgl. Häußling 2014: 14).

Es gibt sehr unterschiedliche Typologien zur Analyse der technischen Entwicklung und deren Wechselverhältnis mit gesellschaftlichen Prozessen (zu einer Übersichtvgl. Häußling 2014:11-86). Rammert (2003: 296) unterscheidet idealtypisch in fünf Stufen, bei denen sich die Mensch-Maschine-Schnittstellen zugunsten der Roboter im historischen Ablauf verschoben haben; in der fünften Stufe kommt der künstlichen Intelligenz (KI) eine hohe Bedeutung zu:

- passive Techniken (Werkzeuge),

- aktive Techniken (Maschinen),

- reaktive Techniken (kybernetische Mechanismen als Kombinationen aus Maschinen und Sensorik - demnach wären automatisierte Fahrzeuge der SAE-Stufe 2 den kybernetischen Techniken zuzuordnen),

- interaktive Techniken (Multiagentensysteme, in denen über wechselseitige Abstimmungen situationsadäquat eine Lösung erarbeitet und das System entsprechend gesteuert wird - danach wären automatisierte Fahrzeuge der SAE-Stufen 3 und 4 den interaktiven Techniken zuzuordnen) und

- transaktive Techniken (intelligente Systeme, bei denen Eigen- und Fremdaktionen in eine Gesamtaktion integriert werden, um gegebene Ziel-Mittel-Relationen selbständig reflek- 
tieren und verändern zu können - demzufolge wären automatisierte Fahrzeuge der SAEStufe 5 den transaktiven Techniken zuzuordnen).

Foerster (1993: 357) benutzt den Begriff „Maschine“ auch für Input-Output-Relationen7, die über rein technologische Anwendungen hinausgehen. Danach gibt es zwei Ausprägungen:

- triviale Maschinen, d. h. eindeutige Input-Output-Beziehungen, wonach ein bestimmter Stimulus eine eindeutige und vorhersagbare Wirkung zeigt; das lässt sich auch auf weitere „planbare“ Prozesse beziehen, die „logisch“ und „rational“ verlaufen;

- $\quad$ nichttriviale Maschinen, d. h. uneindeutige Input-Output-Beziehungen, in denen die „Maschine" auf den Input reagiert, um einen nach eigenen Vorstellungen optimierten Output zu erzielen; das sind „autopoietische Maschinen“ oder eben Menschen, deren Verhalten (Output) von den Beobachtenden mit einem mechanischen Denken nicht analysiert werden kann; in diese Kategorie gehört beispielsweise die Entwicklung der KI.

Diese Unterscheidung ist insofern wichtig, da sich die Menschenbilder in den klassischen Technik- und Ingenieurwissenschaften von denen in den dominanten Sozial- und Geisteswissenschaften deutlich unterscheiden. Während bei Ersteren das Menschenbild trivialer Maschinen dominiert (homo oeconomicus), gehen Letztere eher von Vorstellungen aus, die Foerster (1993) als „nichttriviale Maschinen“ bezeichnet (situationsspezifische Reflexivität). Dies zeigt sich insbesondere in den klassischen Technikfolgen- und Rebound-Forschungen, weil dort die Handlungen von Menschen, die von Wissenschaftlerlnnen als nichtrational angesehen werden, als „Fehlverhalten“ (Rebound) eingeordnet werden. Tatsächlich sind Interpretationen und Handlungen aus Sicht der Handelnden vor dem Hintergrund ihrer eigenen Wahrnehmungen und Bewertungen aufgrund ihrer Sozialisationserfahrungen optimierte Handlungen.

Eine weitere Unterscheidung bezieht sich auf die Position im Rahmen eines wirtschaftlich-technologischen Zyklus (wie beispielsweise die Schumpeter- oder Kondratieff-Zyklen) resp. Produktlebenszyklus. Welge (1992: 270) unterscheidet in

- Basistechnologien (Technologien in der Reifephase des Produktlebenszyklus resp. als tragende Technologien eines wirtschaftlich-technologischen Zyklus),

- Schlüsseltechnologien (Technologien, die das Marktwachstum vorantreiben) und

- Schrittmachertechnologien (innovative oder disruptive Technologien, die einen neuen Zyklus einleiten).

Bezogen auf automatisierte und vernetzte Fahrzeuge wären die Basistechnologien alle jene, die in Fahrzeugen der SAE-Klassen 1 und 2 wirksam sind. Schlüsseltechnologien sind beispielsweise jene, die hinter den innovativen Sensorsystemen, den Effizienzsteigerungen und der Dekarbonisierung der Antriebssysteme, der Beschleunigung der Ladedauer für E-Fahrzeuge und hinter Apps stehen, mit denen die Mobilität vielfältiger organisiert wird (MaaS - Mobility as a Service). Die Schrittmachertechnologien befinden sich aktuell in der Entwicklungsphase - sie liegen im Bereich neuer Treibstoffe, der weiteren Effizienzsteigerung von Rechnerleistungen, vor allem aber in der Weiterentwicklung der Künstlichen Intelligenz zur Steuerung autopoietischer Systeme.

7 „Der Ausdruck Maschine bezieht sich in diesem Zusammenhang auf wohldefinierte funktionale Eigenschaften einer abstrakten Grösse, und nicht in erster Linie auf ein System von Zahnrädern, Knöpfen und Hebeln, obwohl solche Systeme jene abstrakten funktionalen Grössen verwirklichen können“ (Foerster 1993: 357). 
Um das Spektrum techniksoziologischer Fragestellungen abzudecken, schlägt Weyer (2008: 11) vier Dimensionen vor, wobei in seinem Ansatz der Technikdeterminismus deutlich überwiegt. In Übersicht 3 wird jedoch auf den Aspekt „Soziale Strukturen von Technik“ verzichtet, weil Weyer darunter ausschließlich den Anwendungsbereich auf der Mikroebene behandelt; er wird daher in den Bereich „Technikfolgen“ integriert. Eine sozial differenzierte Sichtweise ist kein gesonderter Bereich, sondern sie ist auch für die Technikgenese und die Gestaltung der Technik notwendig.

Übersicht 3: Analysebereich der Techniksoziologie

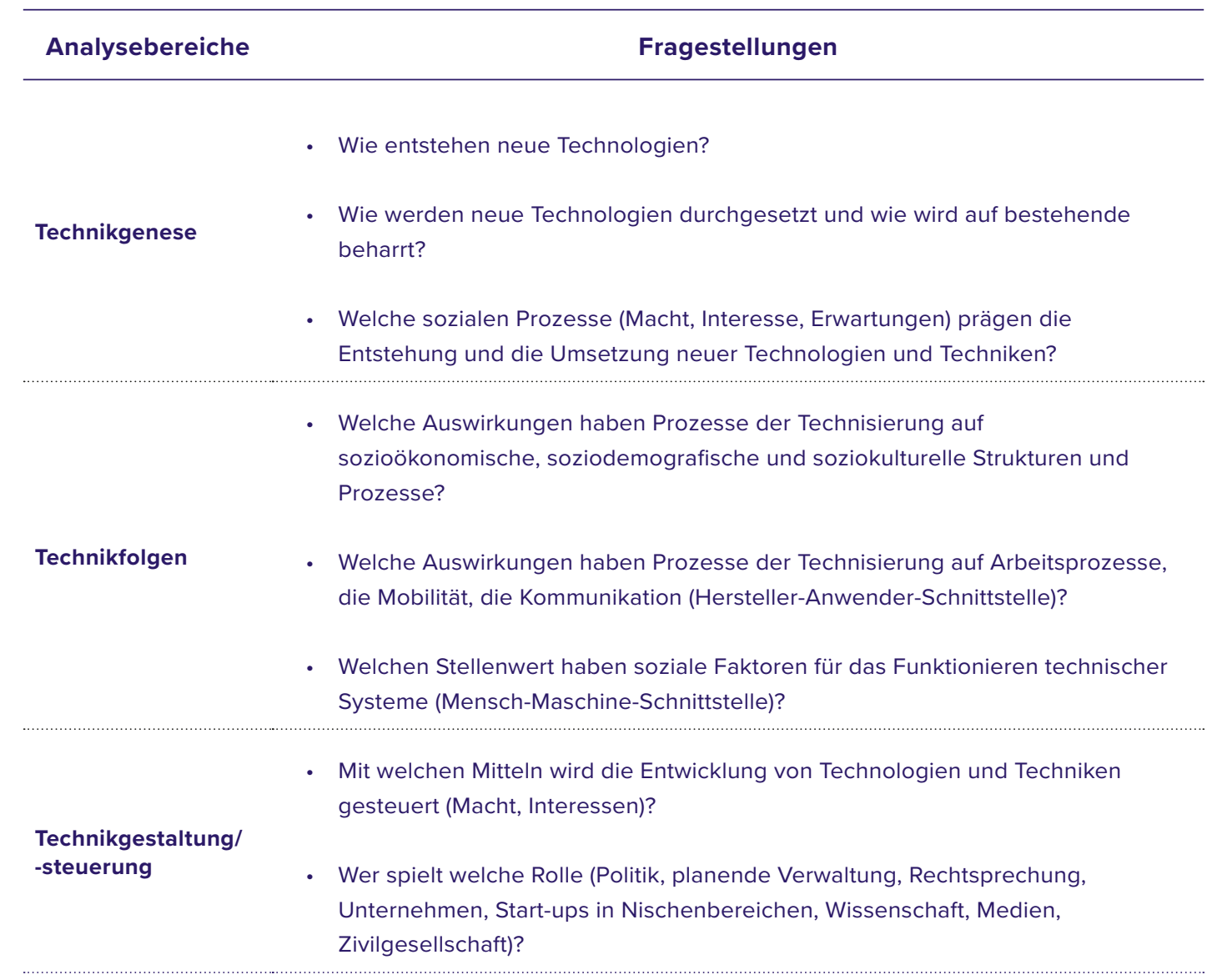

Quelle: eigene Darstellung nach Weyer (2008: 11) und Tarmann (2018: 27f.)

Wendet man diese Analysebereiche auf den avV an, wird deutlich, dass das wissenschaftliche Interesse an den vermuteten (!) Folgen des avV deutlich überwiegt. Der Bereich der Technikgestaltung und -steuerung wird vor allem formaljuristisch behandelt, die unterschiedlichen Interessen der Entwicklung werden eher formal und an institutionellen Ordnungen orientiert thematisiert. Machtverhältnisse resp. vor allem das hohe Interesse der IT-Firmen an der disruptiven Entwicklung des avV, die weniger am Verkehr selbst und der Mobilität orientiert sind, sondern Interesse an den personenbezogenen On-trip-Daten haben, werden kaum einmal behandelt (vgl. Dangschat 2017a, 2019). Sozialwissenschaftliche Studien zur Technikgenese des avV sind bislang eher die Ausnahme. Erste Überlegungen hierzu finden sich bei Manderscheid (2014), Dangschat (2019), Freudendal-Pedersen et al. (2019) sowie Dangschat und Stickler (2020). 


\subsection{ANSÄTZE DER TECHNIKHERSTELLUNG}

Techniken sind nicht plötzlich da und sie fallen nicht vom Himmel. Sie basieren auf Erfindungen (Innovationen), müssen in Systeme eingebaut werden und einen Markt finden. Alle drei Schritte sind mit Interessen sowie Herrschafts- und Machtkonstellationen verbunden. Das bedeutet, dass jede Technik innerhalb eines keineswegs linear und rational verlaufenden Aushandlungsprozesses in einem bestimmten gesellschaftlichen Kontext (Wünsche, Erwartungen, Vorbehalte) durchgesetzt wird. Das Was und Wie, mit welcher Dynamik und zu wessen Vorteil, ist also immer an ein bestimmtes Machtverhältnis und eine Interessenskonstellation in einem „Wirkungsraum“8 und in einen bestimmten Zeitraum gebunden.

\subsubsection{Theorien der Nachfrage und Theorien des technischen Entwicklungsdrucks}

Hinsichtlich der möglichen Treiber eines soziotechnischen Wandels gibt es zwei sich einander gegenüberstehende Schulen: die These der Nachfrage („demand pull theories“) und die des technologischen Entwicklungsdrucks („technology push theories“; vgl. Stefano et al. 2012):

- Mit der Theorie der Nachfrage wird davon ausgegangen, dass Nachfragende über ihr Verhalten den Preis der angewandten Techniken beeinflussen und damit die Dynamik der Marktdurchdringung bestimmen (vgl. Schmookler 1966).

- Mit der Theorie des technischen Entwicklungsdrucks ${ }^{9}$ wird angenommen, dass durch die Möglichkeit, bestehende Techniken zu nutzen, nicht nur innovative Güter und Dienstleistungen bevorzugt wahrgenommen werden, sondern auch Unternehmen und Gebietskörperschaften vor allem naturwissenschaftlich-technische Entwicklungen fördern (vgl. Schumpeter 1942).

Eine Politik, welche der These der Nachfrage folgt, birgt die Gefahr - so der Trend in einigen Branchen (vgl. Hoppmann 2015) -, dass (über)reife Techniken länger als notwendig gefördert werden (vgl. Hoppmann et al. 2013) ${ }^{10}$. Über eine mit nationalen Mitteln unterstützte Nachfrage kann zudem nicht ausgeschlossen werden, dass auch Produkte ausländischer Hersteller gekauft werden. Eine staatliche Technologieförderung würde jedoch gezielt die Innovationen im eigenen Land fördern (vgl. Peters et al. 2012).

8 Während der Zusammenhang zwischen technologischen und gesellschaftlichen Entwicklungen lange meist regional oder nationalstaatlich begrenzt angesehen wurde, zeigen die Entwicklungen im Kontext der Digitalisierung deutlich territorial entgrenzte Zusammenhänge. Das wird mit den Ansätzen der Zweiten Moderne (vgl. Beck et al. 2001; Bonß/Kesselring 2001) und der Organisation von Macht- und Interessenkonstellationen in „scales“ (vgl. Brenner 2019) deutlich.

9 Insbesondere in der angelsächsischen Literatur wird meist der Ansatz mit „technology push“ bezeichnet. Das liegt zum einen am unterschiedlichen Sprachgebrauch des Begriffes „technology“ im Angelsächsischen gegenüber dem Deutschen und ist zum anderen abhängig vom Zeitpunkt, d. h. akademischen Moden. Hier sollen gemäß der eingangs formulierten Definition beide Begriffe unterschieden werden.

10 Ein gutes Beispiel hierfür ist die Debatte um die Kompensation der Verluste in der Automobilindustrie aufgrund der Corona-Pandemie: Soll nur der Kauf von Autos mit postfossilen Antrieben gefördert werden oder auch der von „modernen Verbrennern“? 
Politik und Industrien verfolgen lange - oftmals zu lange - erfolgreiche Entwicklungen, die auf veralteten Basistechniken beruhen. Dieser Sachverhalt hat sich insbesondere seit den 1970er Jahren in den altindustriellen Gebieten Nordamerikas und Europas gezeigt (Stahl und Kohle, Schiffbau, Lederindustrie etc.) - hier war der Erfolg der staatlichen Steuerung und der industriellen Produkte in der Vergangenheit (der 1950er bis in die 1970er Jahre hinein) ein wesentlicher Hinderungsgrund, den politischen und technischen Wandel rechtzeitig und konsequent vorzunehmen (zum Süd-Nord-Gefälle in Deutschland vgl. Friedrichs et al. 1986). Daraus entstanden Pfadabhängigkeiten, die nicht nur das Beharren auf Basistechniken, sondern auch eine Elitenkultur der "Stahl-Barone“ beinhalteten, was sich letztlich aufgrund von (partei)politischen Steuerungen und betrieblichen Entwicklungen in bestimmten Strukturen und Mentalitäten verfestigte (Lock-in-Effekte).

\subsubsection{Sozialkonstruktivistische Ansätze}

Der Sozialkonstruktivismus geht auf die Forschungsarbeiten von Berger und Luckmann (1969) zurück. Demzufolge sind nicht die „Tatsachen“ relevant, sondern die jeweiligen Interpretationen von sozialen Strukturen, Prozessen und Dingen, welche die „Wirklichkeit" schaffen (vgl. Knorr-Cetina 1989). Sozialpsychologische Erkenntnisse zeigen, dass Menschen nicht aufgrund objektiver Tatsachen handeln, sondern immer vor dem Hintergrund eigener Sozialisationserfahrungen und Werte (Habitus) sowie im Rahmen zeitlicher und finanzieller Ressourcen (soziale Lage) gemäß ihren Wahrnehmungen und Bewertungen (Kognitionen; vgl. Bamberg 2004) - daher sieht Foerster Menschen und soziale Gruppen als „nichttriviale Maschinen" an (s. o.).

Dass „Wirklichkeiten“ sozial konstruiert werden, betrifft sowohl die Herstellung (technisches Wissen ist sozial konstruiert) als auch die Anwendung von Technik (subjektive Sinngebung von Technik). Durch den Umgang mit Techniken in der Herstellung und Anwendung werden Techniken als „Problemlöser“ in das jeweilige Handeln eingebaut. Eine häufig übersehene Rolle spielt dabei die Wissenschaft selbst, denn „[...] both science and technology are socially constructed cultures" (Pinch/Bijker 1987: 21).

Dass Sinndeutungen sozial unterschiedlich sind, zeigt sich in verschiedenen Aspekten, die im Kontext des avV relevant sind. Im globalen Maßstab unterscheiden sich die Einschätzungen über die Sicherheit der Fahrzeuge und über den Vorteil der Konnektivität, die Beunruhigung bei Tests im eigenen Umfeld und die Bereitschaft, für Technologien des avV mehr Geld auszugeben, sehr deutlich (siehe Tab. 1).

Tabelle 1: Akzeptanz von Aspekten des automatisierten und vernetzten Verkehrs nach ausgewählten Ländern weltweit (in \%)

\begin{tabular}{|c|c|c|c|c|c|c|}
\hline & DEU & USA & JPN & KOR & IND & $\mathrm{CHN}$ \\
\hline Autonome Fahrzeuge sind nicht sicher. & 45 & 48 & 47 & 46 & 58 & 35 \\
\hline $\begin{array}{l}\text { Die zunehmende Konnektivität der } \\
\text { Fahrzeuge ist ein Vorteil. }\end{array}$ & 36 & 46 & 49 & 56 & 76 & 80 \\
\hline $\begin{array}{l}\text { Beunruhigung über Tests auf öffentlichen } \\
\text { Straßen im eigenen Wohnumfeld }\end{array}$ & 46 & 51 & 41 & 48 & 57 & 32 \\
\hline $\begin{array}{l}\text { Anteil derer, die nicht bereit sind, für Tech- } \\
\text { nologien des avV mehr Geld zu bezahlen. }\end{array}$ & 41 & 34 & 30 & 11 & 8 & 7 \\
\hline
\end{tabular}


Aber auch in Europa gibt es Unterschiede zwischen den Nationalstaaten (siehe Tab. 2): Insbesondere in Deutschland und Österreich sind die Vorbehalte gegenüber der Datensicherheit hoch und der verbesserten Konnektivität können nur Wenige etwas Positives abgewinnen.

Tabelle 2: Akzeptanz von Aspekten des automatisierten und vernetzten Verkehrs nach ausgewählten Ländern in Europa (in \%)

\begin{tabular}{|c|c|c|c|c|c|c|c|}
\hline & AUT & DEU & FRA & ITA & GBR & BEL & ESP \\
\hline Autonome Fahrzeuge sind nicht sicher & 42 & 45 & 38 & 25 & 49 & 50 & 33 \\
\hline $\begin{array}{l}\text { Die zunehmende Konnektivität der } \\
\text { Fahrzeuge ist ein Vorteil. }\end{array}$ & 33 & 36 & 42 & 63 & 49 & 54 & 55 \\
\hline $\begin{array}{l}\text { Beunruhigung über Tests auf öffentlichen } \\
\text { Straßen im eigenen Wohnumfeld }\end{array}$ & 42 & 46 & 40 & 39 & 52 & 40 & 48 \\
\hline $\begin{array}{l}\text { Anteil derer, die nicht bereit sind, } \\
\text { mehr als } 400 € \text { für eine verbesserte } \\
\text { Sicherheit zu zahlen. }\end{array}$ & 64 & 71 & 76 & 69 & 68 & 72 & 64 \\
\hline $\begin{array}{l}\text { Anteil derer, die nicht bereit sind, } \\
\text { mehr als } 400 € \text { für ein verbessertes } \\
\text { Infotainment zu zahlen. }\end{array}$ & 86 & 84 & 84 & 79 & 76 & 88 & 78 \\
\hline $\begin{array}{l}\text { Anteil derer, die nicht bereit sind, } \\
\text { mehr als } 400 € \text { für eine verbesserte } \\
\text { Autonomie zu zahlen. }\end{array}$ & 66 & 67 & 70 & 59 & 61 & 70 & 62 \\
\hline $\begin{array}{l}\text { Anteil derer, die nicht bereit sind, } \\
\text { mehr als } 400 € \text { für eine verbesserte } \\
\text { Konnektivität zu zahlen. }\end{array}$ & 76 & 79 & 79 & 74 & 70 & 80 & 69 \\
\hline $\begin{array}{l}\text { Eine verbesserte Konnektivität ist ein } \\
\text { Vorteil. }\end{array}$ & 33 & 36 & 42 & 63 & 49 & 54 & 55 \\
\hline $\begin{array}{l}\text { Vorbehalte, dass biometrische Daten } \\
\text { gesammelt und mit externen Partnern } \\
\text { ausgetauscht werden. }\end{array}$ & 62 & 62 & 54 & 36 & 54 & 44 & 49 \\
\hline
\end{tabular}

Quelle: Deloitte (2020)

Solche Ergebnisse aus internationalen Umfragen, die fast ausschließlich von den großen, international tätigen Beratungsfirmen durchgeführt werden, haben eine hohe mediale und politische Wirkung. Aus sozialwissenschaftlicher Sicht sind sie jedoch nahezu bedeutungslos resp. bestätigen sie immer wieder bekannte grobe Tendenzen. Zum einen sind solche Daten stark abhängig von der Qualität der Erhebungen: Wurden sie wirklich repräsentativ für das jeweilige Land (sozial und räumlich differenziert) erhoben? Zum anderen werden nur Durchschnittswerte und keine Informationen über unterschiedliche Bewertungen zwischen sozialen Gruppen und siedlungsstrukturellen Gegebenheiten (z. B. Stadt-Land) vermittelt. Solche Studien dienen vor allem der Akquise von Beratungsaufträgen, weniger jedoch wirklicher Erkenntnis über Vorbehalte und Offenheiten.

Ein anderer Zugang kommt aus der Psychologie, wobei erklärt wird, wie „Menschen an sich“ (im Sinne eines menschlichen Grundmusters) Entscheidungen treffen. Jing et al. (2020) haben in einer Sekundäranalyse aus über 75 internationalen Studien zur Akzeptanz automatisierter Fahrzeuge, die zwischen 2013 und 2019 erschienen sind, die wesentlichen Faktoren herausgearbeitet, die über die Akzeptanz des avV entscheiden. Das sind zum einen Persönlichkeitsmerkmale (Vertrauen, erkennbarer Nutzen der Technologie, Bequemlichkeit der Nutzung und 
soziale Kontrolle) und zum anderen Merkmale und Auswirkungen, welche die automatisierten Fahrzeuge selbst betreffen (Sicherheit des Fahrens, finanzielle Rahmenbedingungen als generelle Merkmale und Datensicherheit, persönliche Sicherheit, Erfüllen der Mobilitätsbedürfnisse, Umweltfreundlichkeit, Fahrkomfort, Identifikation mit dem Fahrzeug und die Bewertung der Fahrzeit als weitere Merkmale).

Aber auch hier gibt es zwei grundsätzliche Schwächen: Es werden erstens Aussagen aus unterschiedlichen technischen, kulturellen, räumlichen und politisch-planerischen Kontexten zusammengefasst und als „menschliche Akzeptanz" analysiert, ohne Probleme der Übertragbarkeit und Kontextabhängigkeit zu reflektieren. Das ist insofern bedeutsam, weil in den meisten Studien von vollautomatisierten Fahrzeugen der SAE-Stufe 5 , $\mathrm{d}$. h. von bislang nicht vorhandenen Bedingungen, ausgegangen wird. Zweitens wird nicht nach sozialen Gruppen oder siedlungsstrukturellen Typen unterschieden, was vor dem Hintergrund zunehmender gesellschaftlicher Ausdifferenzierung notwendig wäre.

\subsection{INTEGRIERENDE ANSÄTZE}

Ziel der „integrierenden“ Ansätze ist, sowohl den Technik- als auch den Sozialdeterminismus zu überwinden und die Entwicklung der Technologie/Technik in unmittelbarem Zusammenhang (als „seamless web“) mit gesellschaftlichen, d. h. naturwissenschaftlichen, ökonomischen, politischen, sozioökonomischen und soziokulturellen Bedingungen sowie deren Entwicklung zu sehen. In kritischer Distanz zu den einseitigen Sichtweisen wurden seit den 1980er Jahren - durchaus auch untereinander abgrenzend - unterschiedliche Ansätze entwickelt (vgl. Häußling 2014: 226-278). Ein wesentlicher Teil basiert auf einer konstruktivistischen Sichtweise (vgl. Berger/Luckmann 1969). Demnach gibt es keine „objektive Wirklichkeit“, sondern Menschen erzeugen mit Hilfe ihrer Sinnesorgane, mit ihren jeweiligen kognitiven Fähigkeiten und Interpretationsmustern jeweils „subjektive Wirklichkeiten“, die handlungsleitend sind. Innerhalb des Konstruktivismus gibt es jedoch unterschiedlich radikale Sichtweisen (vgl. Knorr-Cetina 1989).

Demzufolge ist Technik nicht wertfrei, ist nicht „plötzlich da“, kennt keinen einzelnen Erfinder, hat immer einen Orts- und Zeitbezug (= sozialer Kontext aus Machtrelationen und Interessenskonstellationen) und ist immer dynamisch zu betrachten. Technische Produkte sind also immer das Ergebnis von Aushandlungsprozessen sehr unterschiedlicher AkteurInnen, wobei die Marktdurchdringung nur ein Indikator ist. Bereits im Vorfeld von Prototypen werden Bilder über (nicht) wünschbare Zukünfte entwickelt, werden Sachzwanglogiken formuliert sowie Argumente und Institutionen aus dem Diskurs ausgeblendet.

Bemerkenswert ist, dass die Rolle der Wissenschaft bei der Konstruktion von „Fakten“ nur bei wenigen Ansätzen berücksichtigt wird. Ausnahmen bilden die wissenssoziologischen Ansätze des „Edinburgh Strong Programme“ (ESP; vgl. Bloor 1999) und das „Empirical Programme of Relativism" von Collins (1981). Im Gegensatz zum amerikanischen Soziologen Robert K. Merton, der naturwissenschaftliche Erkenntnisse als eindeutig betrachtet, sieht der Ansatz des ESP auch diese Erkenntnisse als soziale Konstruktion an. Daraus entstehe die Notwendigkeit, die Interessenskonstellationen hinter der Durchsetzung naturwissenschaftlicher „Wahrheiten“ zu betrachten.

Collins (1981) kritisiert die Ansätze, bei denen sich die Wissenschaftlerlnnen nur mit "der Welt da draußen" auseinandersetzen, aber die eigene Rolle negieren. Er betrachtet die innerwissenschaftlichen Aushandlungsprozesse in drei Stufen. Interessant ist, dass er auch den naturwissenschaftlichen Untersuchungsanordnungen eine „interpretative Flexibilität“ zuordnet, die insbesondere für die Kommunikation und Wirkmächtigkeit der Ergebnisse relevant ist (Reputation, rhetorische Fähigkeiten). Im zweiten Schritt setzt sich dann eine Sichtweise durch, u. a. auch, 
weil alternative Perspektiven nicht mehr weiterverfolgt werden ${ }^{11}$. Im dritten Schritt werden diese "Erkenntnisse“ Gegenstand politischer Entscheidungen und gesellschaftlicher Überzeugung ${ }^{12}$. Aber auch hier wird nicht zwischen den Natur- und Technikwissenschaften auf der einen und den Sozial- und Geisteswissenschaften auf der anderen Seite unterschieden, denn zwischen innen verlaufen nach wie vor starke Schließungsprozesse (beispielsweise über die Akzeptanz unterschiedlicher Methoden resp. die Bedeutung mathematischer Logik).

\section{Science and Technology Studies (STS)}

Aufgrund eines zunehmend dynamischen und tiefgreifenden sozialen Wandels ist mit der Science and Technology (STS)-Forschung ${ }^{13}$ seit dem Ende der 1980er Jahre eine neue Sichtweise in der Techniksoziologie und ein hochgradig interdisziplinäres Forschungsfeld entstanden (vgl. Bijker et al. 1987). Durch die Erweiterung um Wissenschaftsphilosophie, Technikgeschichte und Techniksoziologie wurden neben den quantitativen Methoden auch qualitative angewandt (ethnografische Methoden, Interviews, Diskursanalyse etc.; vgl. Beck et al. 2012: 11). Mit einer zunehmenden Orientierung an den Herstellungsbedingungen von Technologien und Technik kamen auch noch Steuerungs- und Governanceanalysen hinzu.

Die zentrale sozialkonstruktivistische These besagt, dass sowohl Wissenschaften als auch Technologien und Techniken sozial konstruierte Kulturen sind (vgl. Pinch/Bijker 1987: 21). In diesem Zuge wird der Sinn von ort- und zeitungebundener Erkenntnis bezweifelt - wie es die Naturwissenschaften nahelegen - und auch die Rationalität wird als "relativ zu einer umgebenden Kultur und relativ auf die Gemengelagen, in denen sich behaupten lässt, rationales zu finden und in denen sich rationales behaupten muss", gesehen (Beck, S., et al. 2012: 224).

Aktuell stehen die neuen Herausforderungen durch die KI und ihre Auswirkungen auf die Mensch-Maschine-Schnittstellen im Mittelpunkt des Interesses, was insbesondere bei der Industrie 4.0 und dem Internet der Dinge (IOT) bedeutsam wird. Letztlich ist aber die Geschwindigkeit und Tiefe des technologischen Wandels nicht zuletzt eine politische Frage.

\section{Large Technology Systems (LTS)}

Aus sozialkonstruktivistischer Sichtweise und innerhalb der des STS-Ansatzes beschäftigt sich der Technikhistoriker Hughes (1987) mit technischen Großsystemen (Large Technology Systems - LTS). Um funktionsfähig zu sein, bestehen technische Systeme aus „nahtlos miteinander verbundenen Elementen“ („seamless web“) wie physische Artefakte, Organisationen, natürliche Ressourcen, wissenschaftliche Elemente und legislative Regelungen. „Groß“ ist ein Techniksystem, wenn es geografisch weit verbreitet ist, wenn es eine lange Wirkungsdauer aufweist, wenn es aus komplexen Technologien und Techniken besteht, die koordiniert werden müssen, und wenn der Vernetzungsgrad hoch ist - alle diese Aspekte treffen eindeutig auf das System des automatisierten und vernetzten Verkehrs zu. Ein großes technisches System setzt sich nach Hughes (ebd.: 57-77) in sieben Schritten durch (siehe Übersicht 3).

Da das automatisierte und vernetzte Fahren erst am Beginn der Entwicklungen steht, ist es aus sozialwissenschaftlicher Sicht wichtig, sich mit den „system builder“ zu beschäftigen. Wer sind

11 Beispielsweise, indem man auf batteriegetriebene Fahrzeuge als einzige postfossile Antriebsart setzt.

12 Die aktuellen Analysen der Corona-Pandemie sowie die Maßnahmen zu deren Beherrschbarkeit sind ein gutes Beispiel für die Relevanz dieses Ansatzes.

13 Unter dem Kürzel STS werden bisweilen auch Science, Technology and Society Studies verstanden. 
sie? Wie agieren sie? Welche Stakeholder setzen sich wann mit welchen Argumenten durch? Interessant ist darüber hinaus, wie sich der „technologische Stil automatisierter Mobilität“ entwickelt und verfestigt. Wie verbinden sich die Diskurse des technologischen Transfers mit denen der Akzeptanz? Darüber hinaus ist es für politisch-planerische Entscheidungen wichtig, wie mit den „system builder“ umgegangen wird und wie sichergestellt wird, dass deren Interessen nicht die Ziele einer nachhaltigen Entwicklung gefährden (vgl. Rupprecht et al. 2018).

Übersicht 4: Sieben Stufen der Entwicklung großer Techniksysteme (LTS; nach Hughes 1987)

\begin{tabular}{|c|c|c|}
\hline 1 & Erfindung & soziotechnische Entwicklung, meist als disruptive Erfindung \\
\hline 2 & Entwicklung & $\begin{array}{c}\text { Überführen in eine zukunftsträchtige Technikentwicklung durch ,system } \\
\text { builder‘ unter Berücksichtigung ökonomischer, ökologischer, politischer und } \\
\text { sozialer Aspekte }\end{array}$ \\
\hline 4 & $\begin{array}{l}\text { Technologischer } \\
\text { Transfer }\end{array}$ & $\begin{array}{c}\text { Anpassen der marktfähigen Produkte/Systeme an unterschiedliche zeit- und } \\
\text { raumabhängige Kontexte }\end{array}$ \\
\hline 6 & $\begin{array}{l}\text { Wachstum, } \\
\text { Wettbewerb, } \\
\text { Konsolidierung }\end{array}$ & $\begin{array}{l}\text { Als disruptive Innovation muss sie sich gegen etablierte Techniksysteme und } \\
\text { deren Lock-in-Effekte durchsetzen. }\end{array}$ \\
\hline 7 & Momentum & Phase selbsttragender Produkte/Systeme mit einer festen Etablierung im Markt \\
\hline
\end{tabular}

Quelle: eigene Darstellung nach Häußling (2014: 246-248) und Tarmann (2018: 38f.)

\section{Akteur-Netzwerk-Studien (ANT)}

Die Akteur-Netzwerk-Theorie (ANT) ist ein sozialwissenschaftlicher Ansatz, der seit den 1980er Jahren innerhalb der Science and Technology Studies (STS) entwickelt wurde, um wissenschaftliche und technische Innovationen zu erforschen und zu erklären. Sie gilt als prominenteste Theorie innerhalb der STS, ist allerdings auch umstritten, weil sie der Materialität eine prägende Rolle für die soziotechnischen Konstellationen zuspricht und weil bezweifelt wird, ob sie eine Theorie sei oder doch nur eine (deskriptive) Methode (vgl. Latour 1996, Gad/Bruun Jensen 2010). Die ANT wurde im weiteren Verlauf zu einer umfassenden soziologischen Theorie und Forschungsmethode entwickelt. Sie hat sich als eigenständige Position innerhalb der Soziologie zwischen technischem und sozialem Determinismus etabliert.

Die maßgeblichen theoretischen Beiträge zur Akteur-Netzwerk-Theorie stammen vor allem von Callon (1999), Law (2006) und Latour (2007) und wurden überwiegend im Kontext der STS erarbeitet. Während die frühen Forschungsarbeiten vor allem die Produktion und Funktionsweise von Wissenschaften und Technologien untersuchten, arbeitete sich die Akteur-Netzwerk-Theorie später an den Grundbegriffen der Soziologie ab.

Folgt man den Ansätzen der ANT, agieren Menschen niemals als alleinige Akteurlnnen, sondern immer in Abhängigkeit zu anderen AkteurInnen oder Entitäten. Deshalb ist auch von Akteursnetzwerken die Rede. Zudem werden soziale, technische und natürliche Objekte weder ausschließlich durch natürliche oder technische Faktoren noch ausschließlich durch soziale Faktoren verursacht angesehen, d. h., man versucht, sowohl den Technik- als auch den Sozialdeterminismus zu überwinden. 
Kerngedanke der Akteur-Netzwerk-Theorie ist, dass die Gesellschaft sich aus verschiedenen Elementen zusammensetzt und netzwerkartig verfasst ist. Nach Law (2006: 431) ist die zentrale Erkenntnis der ANT, dass "das Soziale“ nur in Form strukturierter Netzwerke existiert, die aus heterogenen Materialien bestehen. Diese Netzwerke umfassen nicht nur soziale Akteurlnnen, sondern auch materielle Dinge wie technische Artefakte oder diskursive Konzepte (vgl. Peuker 2010).

Das bedeutet, dass man die künftige automatisierte und vernetzte Mobilität weder allein über ihre Stakeholder noch über technologische Erfindungen oder die Narrationen zum avV in politischen Strategien, Medien und der Werbung allein erfassen sollte, sondern es ist notwendig, die gegenseitigen Durchdringungen und die jeweiligen zeit- und raumgebundenen Netzwerke in ihrer Gänze zu analysieren.

Zentraler Untersuchungsgegenstand der ANT ist der Zusammenschluss unterschiedlicher Elemente zu mehr oder weniger kohärenten Akteurlnnen. Von zentralem Interesse sind Assoziationen, die zwischen verschiedenartigen (heterogenen) Entitäten entstehen - also zum Beispiel die wechselseitige Relation zwischen technischer Entwicklung, veränderten Prioritäten der Forschungsförderung und politischen Strategien zur Antriebs-, Verkehrs- und Mobilitätswende. Mit der ANT werden daher Verbindungen aufgezeigt, die ebenso materiell (zwischen Dingen) als auch semiotisch (zwischen Konzepten) bestehen.

Mit der Akteur-Netzwerk-Theorie wird zu erklären versucht, wie sich materiell-semiotische Netzwerke bilden, um als Ganzes zu handeln (z. B. ist der automatisierte Verkehr sowohl ein Netzwerk als auch ein Akteur, und für manche Zwecke agiert er als eine einzige Entität). Als einen Teilaspekt hiervon betrachtet die ANT explizite Strategien, die dazu dienen, verschiedene Elemente zusammen in ein Netzwerk zu integrieren, damit sie nach außen hin als ein kohärentes Ganzes erscheinen.

Die Netzwerke sind jedoch nicht an sich kohärent und können Konflikte enthalten, weil unterschiedliche Interessen einfließen und unterschiedliche Narrationen erzeugt werden (z. B. können Konflikte zwischen den Fahrzeugherstellern und den staatlichen Zulassungsstellen resp. Akzeptanzprobleme in der Bevölkerung gegenüber automatisierten Fahrzeugen entstehen; vgl. Jing et al. 2020); sie treten immer im Rahmen des Aushandelns von Interessen unterschiedlichen Gewichts auf. In Abbildung 1 sind die unterschiedlichen Ansätze der Techniksoziologie grafisch verortet.

Abbildung 1: Analytische Ansätze der Techniksoziologie

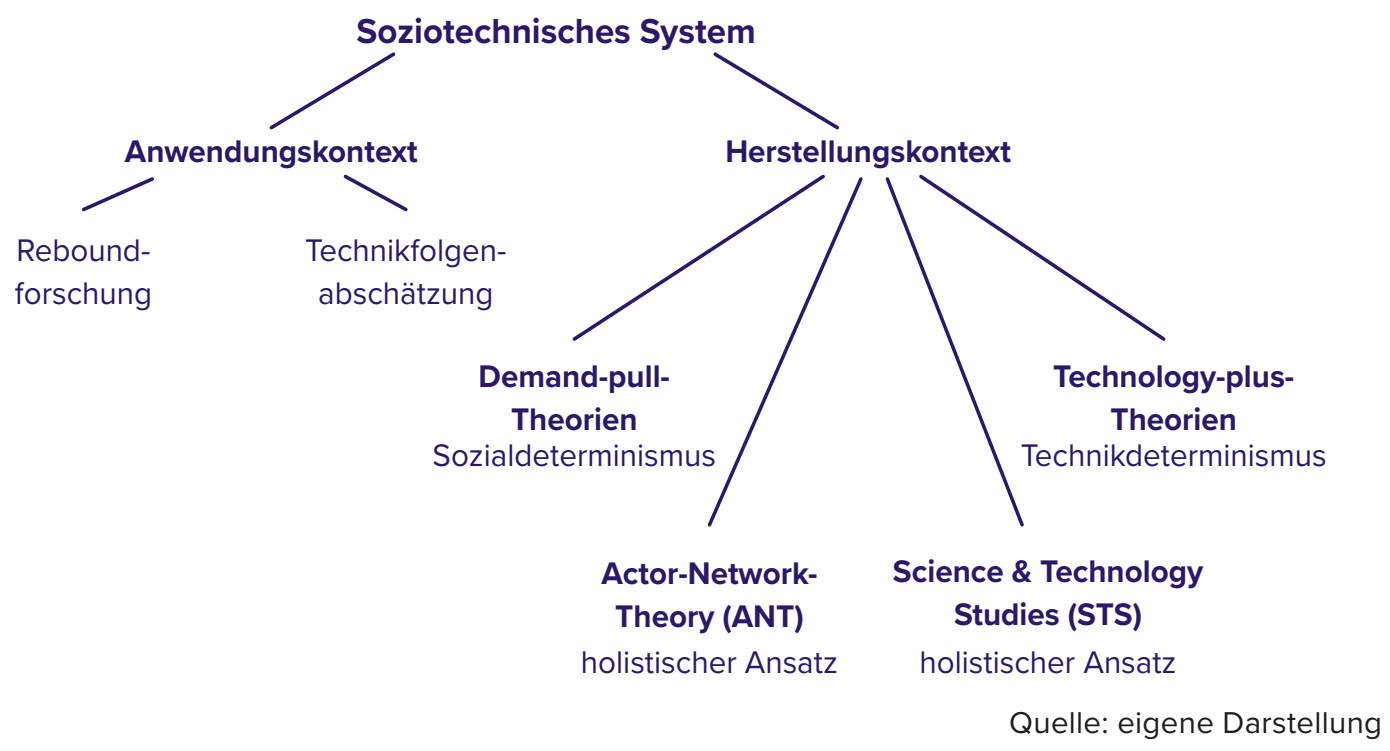




\section{DER ANSATZ VON GEELS - UND WARUM ER ZU KURZ GREIFT}

"[Technological transitions] [...] do not only involve changes in technology, but also changes in user practises, regulation, industrial networks, infrastructure, and symbolic meaning or culture" (Geels 2002: 1257).

Der niederländische STS-Forscher Frank W. Geels setzt sich mit technologischen Innovationen im Kontext soziotechnologischer Transitionen auseinander. Er grenzt sich von denjenigen techniksoziologischen Ansätzen ab, nach denen Technologie keine Eigenschaften hätte, keine Macht und eben "nichts tue“. Dazu entwickelte er in unterschiedlichen Forschungskontexten das Konzept des „technologischen Regimes“ von Nelson und Winter (1982) zu einem soziotechnischen Regime weiter, indem er den Kreis der Akteurlnnen erweiterte und eine größere Aufmerksamkeit auf deren Fertigkeiten, Ressourcen und Interessen legte.

Auch Kanger et al. (2018: 48) distanzieren sich von den reinen Anwendungsansätzen von Techniken und Technologien. Diese seien in der Regel nur auf einzelne Gruppen gerichtet, die zudem meist lediglich in „early adopters" oder „laggards“ eingeteilt werden. Diese Modelle seien darüber hinaus statisch und würden demnach die Wechselseitigkeit von Herstellung und Anwendung, also die "co-creation“ ausblenden. Im Weiteren wird zumindest implizit von einem linearen Ablauf des Wandels ausgegangen und werden jene Entwicklungsprozesse und Auseinandersetzungen ausgeblendet, welche die politische Kultur, die Wertvorstellungen, die politische Maßnahmen und das Verhalten der Anwendenden des soziotechnischen Systems prägen (vgl. Geels 2004).

„Technological diffusion“ wird in diesem Ansatz verstanden

„[...] as a process of co-construction of the entire configuration of sociotechnical systems, including the focal technology and the environments in which it will function" (Kanger et al. 2018: 48).

Die Autoren gehen insofern klassisch systemtheoretisch vor, indem sie in das (Sub-)System („technology“) und ein Umfeld unterscheiden, in dem Technik hergestellt und angewendet wird. Darauf aufbauend hat ihr Ansatz nach eigener Einschätzung drei Vorteile (vgl. ebd.: 48f.):

1. Es werden nicht nur die Anwendenden der Technologien berücksichtigt, sondern auch andere soziale Gruppen.

2. Es wird der Blick darauf geöffnet, dass die Eigenschaften soziotechnischer Systeme nicht von vornherein bekannt sind, sondern dass sich Märkte, deren Steuerung, Infrastrukturen, Akteurlnnen, Präferenzen und Symbole erst im Verlauf der Marktdurchdringung herausbilden.

3. Es wird dadurch sichtbar, dass soziotechnische Prozesse unterschiedliche Verläufe nehmen können, weil die Richtung und Intensität der Innovationen durch die Auseinandersetzungen zwischen den „system builder“, bestimmt werden.

Konkret gehen hinsichtlich der Verbreitung von Innovationen Kanger und seine Ko-Autoren von fünf relevanten Dimensionen „gesellschaftlicher Einbettung“ aus (ebd.: 49f):

1. Einbettung in das Umfeld der "user“ (im weiteren Sinne): Sie gehen dabei über die FahrerInnen von Pkws hinaus, indem sie beispielsweise auch andere Verkehrsteilnehmende, 
den Kraftfahrzeughandel, die Medien, Polizei, Politik und planende Verwaltung einbeziehen, um die neuen Technologien in professionelle und private Alltagsroutinen einbringen zu können ${ }^{14}$. Das erfordere sowohl „kognitive Arbeit“ und „symbolische Arbeit“ als auch „praktische Arbeit“"15.

2. Einbettung in das unternehmerische Umfeld: Dieser Bereich richtet sich auf die industrielle Entwicklung, die Entwicklung neuer Geschäftsmodelle, Angebots- und Verteilungsketten sowie Reparaturwerkstätten. Interessant ist hierbei das Augenmerk auf Innovationen, die von neuen Marktteilnehmenden ausgehen und den Niedergang der traditionellen Strukturen bewirken (hier nehmen sie Bezug auf Schumpeter).

3. Kulturelle Einbettung: Die Artikulation positiver Diskurse, Narrationen und Visionen erzeugen eine kulturelle Legitimation und befördern die gesellschaftliche Akzeptanz der neuen Techniken. Negative Diskurse über traditionelle und positive über neue Techniken beeinflussen Verbraucherpräferenzen und die politische Unterstützung. Die kulturelle Einbettung ist demnach ein widersprüchliches und umkämpftes Feld, weil hier unterschiedliche Interessen aufeinanderstoßen.

4. Regulatorische Einbettung: Hier steht vor allem die Rolle des Staates (Politik und planende Verwaltung) im Mittelpunkt, welche durch das Setzen von Standards und Verordnungen die Produktion, den Markt und die Anwendung von Techniken (mit)gestalten.

5. Einbettung in eine transnationale Gemeinschaft: Die internationale Einbettung fördert durch das wechselseitige Lernen den Austausch von Erfahrungen, die Konsultation und das Aushandeln. Ein Konsens über Standards unterstützt eine gemeinsam geteilte Sichtweise auf Technologien ${ }^{16}$.

Kanger et al. (2018) übersehen mit ihren fünf Dimensionen der Einbettung jedoch, dass diese unterschiedlich gelagert sind. Rein funktional bilden die steuernden Rahmenbedingungen der Politik (und planenden Verwaltung) eine Reihe von Schnittstellen der Förderung und Reglementierung mit dem unternehmerischen Feld. Die drei anderen Bereiche der Einbettung liegen auf völlig anderen Ebenen.

14 Die Autoren verfolgen - ohne dieses explizit auszuführen - den Ansatz des „akteurzentrierten Institutionalismus" (vgl. Mayntz/Scharpf 1995), wonach Menschen immer in Akteurskonstellationen handeln. Ihre Zielsetzung und Interessen richten sich nach den externen Vorgaben des jeweiligen institutionellen Kontextes und der eigenen dauerhaften Handlungsorientierung sowie nach situativen Handlungsmotiven. Häußling (2014: 250) unterstreicht die Bedeutung dieses Ansatzes für die technologiepolitische Steuerbarkeit großer technischer Systeme, d. h. der Technikentwicklung selbst und der Steuerung von Infrastruktursystemen.

15 Interessant ist, dass hier die Rolle der (interpretativen) Wissenschaft von den Autorlnnen ausgeblendet wird.

16 Bei der Darstellung dieser Ebene beziehen sich Kanger et al. (2018) auf die Perspektive eines historischen Transnationalismus, der Automobilität und nachhaltiger Entwicklungen. Aus meiner Sicht ist die Darstellung einseitig positiv und daher realitätsfern, weil gerade im Wettstreit um die Ökonomisierung technischer Innovationen Nationalstaaten und Unternehmen in heftiger Konkurrenz zueinander stehen und sich eher abschotten resp. allenfalls kooperieren, um die Konkurrenz positiver zu gestalten. Selbst der (unterschlagene) Bereich der Wissenschaften ist kaum von den Bemühungen um Konsens und Kooperation gekennzeichnet. 
Die transnationale Ebene ist keine eigene Dimension, sondern stellt lediglich eine Maßstabsverschiebung auf die internationale Ebene dar (vgl. den „Scales“-Ansatz von Brenner 2019). Das Feld der kulturellen Einbettung beschreibt die Art der Auseinandersetzung, die Argumente und die "Waffen“, mit denen auf jeder der Ebenen und zwischen unterschiedlichen Ebenen „gekämpft" wird. Dazu gehört auch die regulatorische Einbettung, die ebenfalls von unterschiedlichen Kulturen und Machtkonstellationen geprägt ist.

Schließlich kann die Dimension der „User“ nicht von den anderen Dimensionen getrennt werden, weil gerade nach dem Selbstverständnis ihres eigenen Ansatzes der Prozess der technischen Entwicklung von sehr unterschiedlichen Akteurlnnen beeinflusst wird - in der Politik, in den Unternehmen, auf lokaler, nationaler und internationaler Ebene. Die wechselseitige Beeinflussung erfolgt immer und ausschließlich im Rahmen der an Ort und Zeit gebundenen kulturellen Einbettung.

Aufbauend auf verschiedenen Arbeiten aus seinen unterschiedlichen Forschungskontexten der „Multi-Level Perspective on Technology Transitions“ (MLP; vgl. Kemp 1994, Rip/Kemp 1998) entwickelte Geels (2002: 1260-1263) einen Mehrebenenansatz, den er in verschiedenen Publikationen auf die Transformation unterschiedlicher Technologien und Techniken angewendet und dabei sein Modell weiterentwickelt hat (vgl. Geels 2004, 2005, 2006, 2011, 2012; Geels et al. 2012). Auf der Landschaftsebene unterscheiden Geels und Schot (2007: 404) anhand der Dynamik von vier Aspekten - Frequenz, Amplitude, Geschwindigkeit und Umfang - vier Typen der Veränderung: normale Veränderung (langsam und inkrementell), spezifischer Schock (plötzliche starke Veränderung in nur wenigen Dimensionen, die zum Wiedereinpendeln in den ursprünglichen Zustand führt), disruptive Veränderung (unregelmäßige, langsame Veränderungen, die nur wenige Dimensionen betreffen ${ }^{17}$ ) und "Lawine“ (das Ausmaß, das Tempo und die Reichweite der Veränderung sind sehr hoch).

Im Mittelpunkt der Überlegungen steht das soziotechnische Regime auf der Mesoebene, das aus sieben Elementen besteht: Technologie, Infrastrukturen, industrielle Netzwerke und unternehmerische Strategien, technisch-wissenschaftliches Wissen, sektorale Politiken, Märkte und Praktiken der Anwendenden sowie die symbolische Bedeutung (siehe Abb. 2). Diese Regimes stehen jedoch im Rahmen eines konkreten territorialen Kontexts (das kann eine Industrieregion sein, meist wird darunter jedoch ein Nationalstaat verstanden) und in eine (globale) Landschaft der (langsamen) Entwicklungen (Makroebene) eingebunden.

Auf der Mikroebene werden nach Kemp et al. (2001) technologische Nischen verortet, die als „Räume der Inkubation“ für radikale Erneuerungen angesehen werden und aus denen die technischen Innovationen hervorgehen.

In einer Reaktion auf unterschiedliche Kritiken an seinem Ansatz hat Geels (2011) sein Modell spezifiziert. Nun räumt er ein, dass die "soziotechnischen“ Landschaften auf der Makroebene als „exogener Kontext“ fungieren und von dort sehr wohl auch ein Druck auf das soziotechnische Regime auf der Mesoebene ausgehen kann, der Neues ermöglicht. Das soziotechnische Regime sieht er nach wie vor in einem „dynamisch-stabilen Gleichgewicht“, das auch die Nischen beeinflusst (durch Erwartungen an deren Innovationen und in Netzwerke eingebunden ${ }^{18}$ ).

17 Ich finde die Bezeichnung „disruptiv“ für diesen Veränderungstyp nicht angemessen, weil im üblichen Verständnis von Technikforschung damit ein fundamentaler, sehr plötzlicher Wandel verstanden wird - also eher das, was sie als „Lawine“ bezeichnen.

18 In diesen Bereich gehören vor allem auch die Förderungspolitik transnationaler, nationaler, regionaler öffentlicher Einrichtungen sowie unternehmerische Förderungen der universitären und außeruniversitären Forschung, die hier unerwähnt bleiben. 
Abbildung 2: Eine dynamische Mehrebenenperspektive technologischer Transformationen

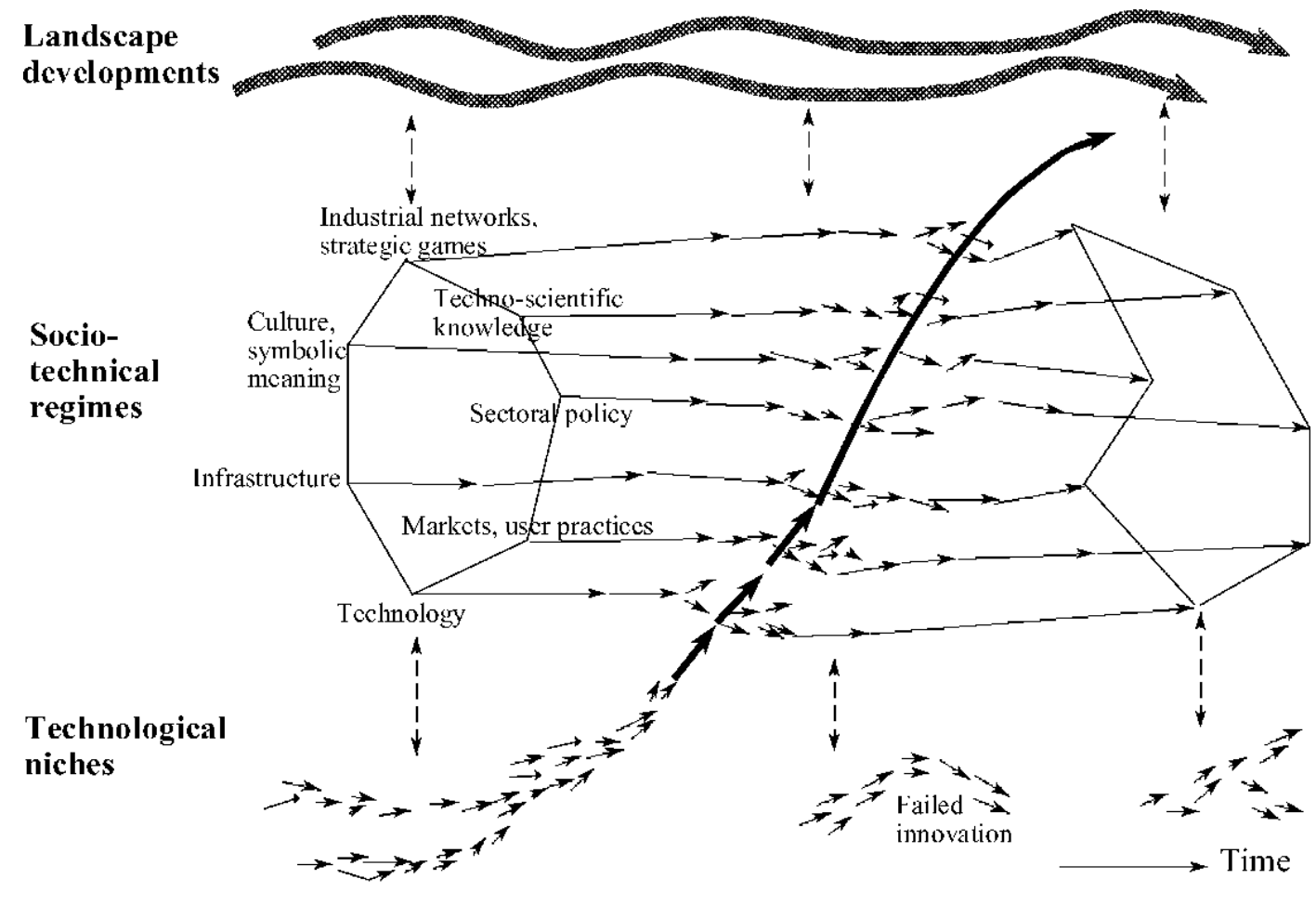

Quelle: Geels (2002: 1263)

Für die Anwendung dieses Modells auf den avV muss bezweifelt werden, ob die Rolle der Nischen und die „Landschaft der Entwicklungen“ von Geels richtig eingeschätzt werden. Für die ersten Fahrassistenzsysteme wie ABS mögen technologische Innovationen „bottom-up“ aus den Nischen noch relevant gewesen sein, aber für den Entwicklungsdruck für hoch- und vollautomatische Fahrzeuge ist vor allem der Top-down-Effekt aus den Entwicklungsschwerpunkten der globalen Landschaft verantwortlich (vgl. Berkhout et al. 2004: 62). Von hier wirken sich die Globalisierung, der Wettbewerb unter den großen Automobilherstellern um Marktführerschaften auf unterschiedlich dynamischen Teilmärkten, branchenfremde Unternehmen aus dem IT-Bereich, politische Einflüsse auf die (nationale) Forschungsförderung im Kontext des Standortwettbewerbs, die Bekämpfung des Klimawandels sowie eine zunehmende Kritik an der Automobilität auf die soziotechnischen Regimes aus. Das bedeutet nicht, dass einzelne technische Entwicklungen beispielsweise in den Bereichen der Sensorik, des Datenmanagements, der Vernetzung in Start-ups und Fab-labs entstehen, die jedoch rasch in die großen Unternehmen der IT-Branche, der Zulieferindustrie resp. der Fahrzeughersteller integriert und damit Bestandteil des soziotech-nischen Regimes und/oder der „landscape“ werden.

Genus und Coles (2008) kritisieren, dass das Konzept von Geels zu formal und die Aktionen insbesondere der politischen Steuerung unterbelichtet seien. Sie schlagen vor, stärker die im Rahmen der sozialkonstruktivistischen Techniksoziologie entstandenen Ansätze der „Social Construction of Technology“ (SCOT), des Akteur-Netzwerk-Ansatzes (ANT) und des "Constructive Technology Assessment" (CTA) zu berücksichtigen, weil dort die Interessen und Motivationen der Stakeholder explizit berücksichtigt werden (siehe Abschnitt 3.3).

Mit den Ansätzen der „policy mobilities“ und des „mobility transfers“ soll daher im folgenden Abschnitt das Augenmerk stärker auf Aspekte der Macht und Interessen vor allem auf der Makroebene gerichtet werden. Mit diesen Ansätzen wird analysiert, wie Narrationen aus der 
"globalen Landschaft" mit ihren unterschiedlichen regionalen und kulturellen Kontexten in der Politik und der planenden Verwaltung aufgenommen werden und eine gänzlich neue Dynamik entfalten können. Vor allem auf der Ebene der kulturellen Einbettung werden Narrationen erzeugt, welche die Akzeptanz automatisierter und vernetzter Fahrzeuge erhöhen sollen. Mit diesem Diskurs soll zum einen die Automobilität nicht infrage gestellt und zum anderen ein positives Beispiel für die Digitalisierung installiert werden (vgl. Dangschat 2019).

\title{
5. „MOBILITY TRANSFERS“ UND „POLICY MOBILITIES“: WARUM DER ENTSTEHUNGSKONTEXT UND DIE ERZEUGUNG VON BILDERN FÜR EINE KRITISCHE ANALYSE SO WICHTIG SIND
}

\begin{abstract}
Die Adressaten und Verwender wissenschaftlicher Ergebnisse in Politik, Wirtschaft, und Öffentlichkeit werden „[...] zu aktiven Mitproduzenten im gesellschaftlichen Prozeß der Erkenntnisdefinition - eine Entwicklung von ,hochgradiger Ambivalenz“" (Beck 1987: 250)
\end{abstract}

Im Gegensatz zu den dominanten techniksoziologischen Ansätzen der Anwendung von Technologien und Techniken sowie den „bottom-up“ gedachten technischen Innovationen setzen sich aktuelle Mehrebenenanalysen in der Stadt- und Regionalforschung vor allem mit den Strukturen, Prozessen, Interessen und Machtstrukturen auf der (globalen) Makroebene und mit der Herstellung von Narrationen künftiger Entwicklung auseinander (vgl. Farias/Bender 2010). Ausgehend von einer Kritik an neoliberalen Tendenzen der eigenständigen Entwicklung großer Metropolen im Standortwettbewerb unterhalb der Ebene der Nationalstaaten wurde innerhalb der „Policy transfer“ (PT)-Forschung die Frage gestellt, wie Politiken (neoliberal vs. deliberativ), Verwaltungsorganisationen („new public management“), Zielsetzungen und Strategien aus einem politischen System in ein anderes übertragen werden (vgl. Dolowitz/ March 2000). Ausgehend von noch stark strukturalistischen Positionen wurde analysiert, wie und warum ein politischer Transfer beispielsweise zwischen der lokalen/regionalen und der nationalstaatlichen Ebene funktioniert (vgl. Evans/Davies 1999). Meist wurden Politiktransfers in einem Land oder einem eher spezifischen Kulturkreis (der „Ersten Welt“) betrachtet. Dazu wurden vor allem die einen Transfer begünstigenden und behindernden Faktoren herausgearbeitet. Als Reaktion auf die anfangs ausschließlich positivistische Auffassung rationaler Entscheidungen wurden innerhalb des PT-Ansatzes zunehmend auch subjektive Prozesse im Politiktransfer berücksichtigt.

Im PT-Ansatz stehen sich zwei (idealtypische) Positionen gegenüber:

- die der Konvergenz aufgrund der Übernahme von (westlichen) Werten, Logiken des Marktes und der Verfasstheit von Demokratien letztlich auch aufgrund der Harmonisierungsbestrebungen internationaler Staatenverbünde und

- die der Divergenz aufgrund von unterschiedlichen Pfadabhängigkeiten, Machtkonstellationen, Diskursen, Lernprozessen und politischen Auseinandersetzungen.

Aktuell geht man eher von der Gleichzeitigkeit beider Tendenzen aus. Die Wahrscheinlichkeit der Konvergenz ist umso höher, je stärker eine Neuausrichtung in eine "große Erzählung" ein- 
gebunden ist. Das dürfte auch für die weltweite Durchsetzung des avV gelten. Zum einen gibt es zahlreiche Belege dafür, dass sich die Automobilität im Sinne der Konvergenz in den Industriestaaten und den Schwellenländern rasch ausbreitet resp. stabilisiert. Die Umsetzung und Akzeptanz wird jedoch unterschiedlich verlaufen - nicht prinzipiell, sondern mit einer unterschiedlichen Dynamik.

Die „Urban-policy-mobilities“ (UPM)-Forschung setzt sich von den Positionen des PT kritisch ab. Dabei werden explizit Parallelen zur These der Netzwerkgesellschaft, zur Zweiten Moderne, zum „mobility turn“, zur Akteur-Netzwerk-Theorie und zum Assemblage-Ansatz hergestellt (vgl. McCann/Ward 2012, Künkel 2015: 8f.; siehe Abschnitt 2.1). Danach werden fixe administrative Ebenen immer durchlässiger und zu „scales“ (vgl. Brenner 1998). Aufgrund bestimmter Mixturen aus technischen und administrativen Praktiken werden neue Räume erschlossen und verständlich, indem Territorien „dechiffriert“ und „neu kodiert“ werden („assemblage“; vgl. Deleuze/Guattari 1992: 699).

Gerade in der zunehmenden Konkurrenzsituation der Metropolen werde eine neue Skalierungspolitik betrieben, indem Institutionen und AkteurInnen aus unterschiedlichen Ebenen in ein strategisches Netzwerk eingebunden werden (vgl. Brenner 2019: 206-233). Dazu wird der Kreis der betrachteten Akteurlnnen über Politik und Verwaltung hinaus deutlich erweitert.

Zudem wird das Augenmerk der Analyse von organisatorischen Strukturen hin zu Diskursen und Machtkonstellationen gerichtet, indem man nachverfolgt, wie sich Ideen, Konzepte und Strategien über "scales“ und die Zeit entwickelt haben (vgl. Peck/Theodore 2010). In diesem Kontext werden auch Elemente einer ethnografischen Politikforschung angewandt (vgl. Wiesner 2003). Es geht der UPM-Forschung über das beschreibende Wie von Strukturen hinaus, um die Frage des „how, why, where and with what effects policies are mobilised, circulated, learned, reformulated and reassembled" (McCann/Ward 2012: 326). Es geht also letztlich um die Frage, wie Strategien von welchen „system builder“ mit welchen Argumenten durchgesetzt und wie globale Politikdiskurse auf andere „scales“ „heruntergebrochen“ werden.

Eine besondere Rolle spielt in diesem Kontext die Wissenschaft als Mediator. Sie erzeugt mit den „vehicular ideas“ eine neue Kategorie von Argumenten innerhalb der Diskurse (vgl. McLennan 2004; vgl. zur Konstruktion von Mobilität: Mincke 2016). Aufbauend auf einer Diagnose gegenwärtiger Herausforderungen werden „problemlösende Vorschläge“ in den Diskurs eingebracht, die ein System in eine wünschenswerte Situation bringen sollen ${ }^{19}$. Peck (2012) verdeutlicht diesen Sachverhalt am Diskurs über die „kreative Stadt“, mit dem Unzulänglichkeiten der politischen und administrativen Steuerung überwunden resp. eine nachhaltige Entwicklung ermöglicht werden kann. Diese Sichtweise ist für die Analyse des avV insofern relevant, weil er seitens der nationalstaatlichen und EU-Politik vor allem als der Löser vielfältiger Probleme des Verkehrs und der Mobilität sowie des Umweltschutzes und der Chancengleichheit dargestellt wird.

Vor dem Hintergrund der bereits aktuellen und künftig absehbaren breiten gesellschaftlichen Bedeutung der Digitalisierung und der darin eingebetteten automatisierten und vernetzten Mobilität erscheint es sinnvoll, auf der Basis eines Mehrebenenmodells die soziotechnische Transformation des Verkehrs mit den Ansätzen des „policy transfers“ (PT) und insbesondere der „policy mobilities“ (UPM) zu analysieren - das steht bislang international noch aus.

19 Ein gutes Beispiel hierfür sind die Arbeiten von Richard Florida (2002) zur "creative class“, die er als Voraussetzung für eine wettbewerbsfähige Stadtentwicklung propagierte und die weltweit aufgenommen wurde (vgl. Dzudzek 2016: 95 ff.). 


\title{
6. DIGITALISIERUNG UND AUTOMATISIERUNG ALS TREIBER DES SOZIOTECHNISCHEN WANDELS?
}

\begin{abstract}
„Die große Transformation beschreibt einen massiven ökologischen, technologischen, ökonomischen, institutionellen und kulturellen Umbruchprozess zu Beginn des 21. Jahrhunderts. [... Dazu bedarf es] „einer besonderen (transformativen) ,Literacy; $d$. h. einer Kompetenz, diese Dimension in ihrem Zusammenspiel zu verstehen, und der Kunstfertigkeit, dieses Verständnis in Beiträge zu einer Nachhaltigen Entwicklung umzusetzen" (Schneidewind et al. 2018: 11).
\end{abstract}

Die Globalisierung ist seit den 1980er Jahren eine „große Erzählung“, die vor allem als Chance kommuniziert wird. Wenn man als Nationalstaat oder Unternehmen die eigene ökonomische Position verteidigen oder ausbauen möchte - so eine weltweit verbreitete These - , sollte man sich dieser Herausforderung proaktiv, rasch und konsequent öffnen. Die Globalisierung hat durch die Vernetzung zu einer zunehmend beschleunigten und weiterreichenden Mobilität von Menschen, Waren, Finanzen, Informationen, Technologien, Ideen, Kulturen und Ideologien geführt, was wiederum Gesellschaften, lokale Bindungen und die Bedeutung der Nationalstaaten relativierte (vgl. Beck 2007, Endres et al. 2016).

Im Zuge der aktuellen Corona-Pandemie und dem daraus resultierenden Lock-down in nahezu allen Industriestaaten wurden sehr strikte Ausgangssperren verhängt, welche die Industrie und den Handel heruntergefahren und überregionale Lieferketten unterbrochen haben. Es entstanden Versorgungsengpässe bei Vorprodukten und Waren, vor allem bei Schutzkleidungen und Grundstoffen für Medikamente. Das wiederum hat nicht nur den Verkehr und die Mobilität zumindest zeitweise komplett verändert, sondern hat auch aufgrund des Bewusstseins über starke überregionale Abhängigkeiten zu einer (weiteren) kritischen Reflexion der Globalisierung geführt.

Die Erzählung der Globalisierung wird seit der Jahrtausendwende von der über die technologiebasierte Digitalisierung überlagert, infolge derer u. a. eine Konkurrenz durch die „smart city" entstand, in der nicht mehr Produktion und klassische Dienstleistungen im Mittelpunkt stehen, sondern Kommunikation, Mobilität und „data management“. Konsequenterweise seien vor allem Technologien und die darauf basierenden Schlüsselindustrien zu fördern, um LifeSciences, künstliche Intelligenz, das Internet der Dinge und die Industrie 4.0 etc. zu entwickeln (siehe Beitrag 20 von Banerjee et al. in diesem Band). Die Erkenntnisse im Umgang mit der Corona-Krise und den damit verbundenen Erfahrungen bezüglich Homeoffice, Telekonferenzen und dem "distant learning" werden zu einer weiteren beschleunigten Digitalisierung von Erwerbsarbeit, Ausbildung, Lernen und Alltagskommunikation führen.

Insbesondere in China, Südkorea und Singapur wird zudem davon ausgegangen, dass der avV wichtig dafür sei, um mit der aktuellen Corona-Krise umzugehen, weil selbstfahrende Fahrzeuge dafür genutzt werden können, den öffentlichen Raum gefahrlos überwachen und ggf. desinfizieren resp. benötigte Güter zur Krankenversorgung rasch und sicher befördern zu können. Zudem seien wegen der nicht vorhandenen Ansteckungsgefahr Pflegeroboter sehr gut für die Versorgung von Covid-19-Patienten geeignet. Von „Start-ups“ entwickelte Prototypen sind dort bereits im Einsatz. Im Gegensatz dazu fahren die Automobilhersteller und Zulieferbetriebe in Europa und Nordamerika ihre Investitionen in die Entwicklung der Automatisierung aufgrund der finanziellen Engpässe durch die Corona-Krise zurück. 
Die vielfältige und weitreichende technologische Transformation der Digitalisierung im Allgemeinen und der (Weiter-)Entwicklung des avV im Besonderen treffen auf Gesellschaften im Umbruch - letztlich auch in Folge von Globalisierung, Digitalisierung und Klimawandel. Sich verschärfende sozioökonomische Ungleichheiten werden durch „Entgrenzungen“ sowie von Freisetzungen aus traditionellen Strukturen mit der Freiheit und dem Zwang zur Selbstorganisation der Biografien überlagert (vgl. Hitzler 1984, Beck 1995). Damit steigen auch die Ansprüche an eine politisch-planerische Steuerung (Produktion von Sicherheit auf Basis zunehmender Unwägbarkeiten). Gleichzeitig verlieren Nationalstaaten ihre Steuerungskraft und ihren Steuerungswillen, was zu Verunsicherungen, insbesondere in der abstiegsbedrohten gesellschaftlichen Mitte, führt.

Auf diese Situation trifft der Diskurs über die Unausweichlichkeit eines avV. Die Anwendung von Technik verlangt jedoch ein spezifisches Wissen und eine hohe Handlungskompetenz. Damit ist die Art, wie eine Technik angewendet werden kann, eine „Verfügungsgewalt“ und damit eine Machtquelle (vgl. Mackenzie/Wajcman 1985). Die Anwendung neuer Technologien und Techniken hat - ebenso wie die Automobilität - bislang immer dazu geführt, bestehende soziale Ungleichheiten zu verstärken und/oder neue hinzuzufügen (vgl. Cudworth et al. 2013, Urry 2012, Dangschat 2019). Im Kontext der zunehmenden elektronischen Kommunikation im Allgemeinen und der Automatisierung und Vernetzung des Verkehrs sowie des Angebots neuer Mobilitätsdienstleistungen im Besonderen, spielt zudem der ausschließende Zugang zu diesen Systemen über digitale Kanäle („digital divide“) eine bislang wenig beachtete sozial selektive und ausgrenzende Rolle (siehe Beitrag 20 von Banerjee et al. in diesem Band).

Neben den immer wieder kommunizierten potentiellen Vorteilen des avV, insbesondere in Form hoch- und vollautomatisierter Fahrzeuge, muss aber auch die Frage erlaubt sein: Wie ist die Idee des automatisierten Fahrens ,in die Welt“ gekommen? Und: Wie ist es dazu gekommen, dass Digitalisierung, Automatisierung und Vernetzung als „unausweichlich“ dargestellt werden, als alternativlos? Im Diskurs über den avV spielen lokale Bedingungen, politisch-planerische Alternativen oder die reservierte Skepsis in der Bevölkerung allenfalls eine untergeordnete Rolle. Hinsichtlich des avV sollte es aber darum gehen, nicht erneut die Siedlungsstrukturen, den öffentlichen Raum und die Verteilung von Standorten den technologischen Bedingungen anzupassen, sondern den avV räumlich und sozial differenziert so umzusetzen, dass er geeignet ist, die notwendigen Antriebs-, Verkehrs- und Mobilitätswenden zu unterstützen (vgl. Rupprecht et al. 2018, Dangschat 2019, Manderscheid 2020 sowie Beitrag 4 von Manderscheid in diesem Band).

Das würde bedeuten, die Diskussion wie folgt sorgfältiger zu unterscheiden:

- nach dem Ausmaß des Automatisierungs- und Vernetzungsgrades, insbesondere für das heterogene und risikoreiche Lange Level 4 (vgl. Mitteregger et al. 2020b),

- $\quad$ nach dem Umfeld, in dem der avV genutzt werden soll (Operational Design Domain ODD; vgl. Mitteregger et al. 2020b: 60-63), was insbesondere die Möglichkeit einschließt, neben den leistungsfähigen Fernstraßen den ländlichen und kleinstädtischen Raum bevorzugt als Testgebiet zu nutzen (siehe Beitrag 6 von Soteropoulos et al. in diesem Band), sowie

- nach sozialer Selektivität, was die unterschiedliche Kaufkraft, die unterschiedlichen Einstellungen zu direkt und indirekt mit dem avV verbundenen Themen beinhaltet (Automobilität, Technikaffinität, Umwelt- und Klimaschutz, Sharing etc.) und den ausgeweiteten „digital divide" betrifft (vgl. Dangschat 2019).

Der Komplex des automatisierten und vernetzten Verkehrs kann sicherlich als ein "großes technisches System" betrachtet werden (siehe Abschnitt 4; vgl. auch Tarmann 2018), das sich 
in Europa im Übergang zur Phase der Entwicklung befindet; allenfalls technische Teilsysteme der schrittweise verbesserten Fahrassistenzsysteme befinden sich in der Phase der Innovation $\left(\right.$ Phase 3) ${ }^{20}$.

Der automatisierte und vernetzte Verkehr wird seitens der Technik- und Ingenieurwissenschaften sowie der Politik auf der EU- und den meisten nationalen Ebenen als ein „sicheres“ Techniksystem dargestellt (vgl. Stickler 2020a), mit dem die Zahl der Unfälle deutlich reduziert wird. Die Sichtweise als deterministische Entlastungsinstanz für soziale Probleme entspricht einem Politikselbstverständnis der „traditionellen Moderne“. Auch wenn die Bewältigung der ersten Phase der Corona-Krise zu einer Stärkung des Vertrauens in den Politikstil des "starken Nationalstaates“ geführt hat, zeigt die aktuelle Entwicklung der „Normalisierung“ deutliche Interessensgegensätze, eine umstrittene Rolle wissenschaftlicher Expertise, das Erstarken des Einflusses „neuer Medien“, einschließlich Verschwörungstheorien und Fake News, d. h., dass wieder die Zweite Moderne als Kontext der politisch-planerischen Steuerung wichtig wird.

Im Weiteren zeigt sich in dieser Entwicklung, dass die tradierten Konzepte politisch-planerischen Handelns nicht mehr greifen und dass es daher neuer Aushandlungsprozesse mit zusätzlichen Akteurlnnen und über unterschiedliche "scales“ hinweg bedarf. Es ist zudem zu erwarten, dass die Orientierung an der immanenten Logik von Naturwissenschaften konflikthaft auf die neuen Akteurlnnen und insbesondere die Nutzenden von automatisierten und vernetzten Fahrzeugen treffen. Es handelt sich dabei um Situationen, die von Foerster (1993) als „nichttriviale Maschinen" bezeichnet werden, weil Wahrnehmungen, Entscheidungen und Prozesse zunehmend nach einer „Eigenlogik“ ablaufen. Hier werden nicht nur die ProduzentInnen und politisch-planerischen Entscheidende vor neue Aufgaben gestellt, sondern auch die Wissenschaften müssen sich ihrer neuen Rolle bewusst sein - das darf sich jedoch nicht darin erschöpfen, die Verantwortung in eine fragwürdige „co-creation“ eines „urban mobility labs“ zu geben.

Folgt man Kesselring (2020) zur Mobilität in der Zweiten Moderne, dann wird künftig der Kontext der Implementierung des avV noch stärker und zunehmend von Unvorhersehbarkeiten und Diskontinuitäten geprägt sein (siehe Abb. 1; vgl. auch Läpple 2011). Dabei werden weiterhin kaum noch Eindeutigkeiten bestehen, sondern zur Problemlösung werden eine Reihe von „Best-Way“-Lösungen nebeneinander entwickelt werden - wie beispielsweise zur Gestaltung der Antriebswende oder von Sharing-Lösungen zur Gestaltung der Mobilitätswende resp. des avV selbst (vgl. Shladover 2016). Das bedeutet für politisch-planerische Lösungen ein gewisses Risiko, die verstärkte Notwendigkeit für ein prozessorientiertes Vorgehen und das Einbeziehen sehr unterschiedlicher Akteurlnnen. Zudem sind die institutionellen Akteurlnnen auf der nationalstaatlichen Ebene nicht die einzigen Steuernden, denn es werden zum einen multinationale politische Entscheidungen als Rahmensetzungen vorgegeben (vor allem der EU), zum anderen sind die Forschungs- und Entwicklungskonstellationen zunehmend international aufgestellt, entziehen sich staatlicher Steuerung, wobei technologische Innovationen und deren Umsetzung häufig auf „Nischen-Akteurlnnen“ basieren (vgl. Kanger et al. 2018).

Mit dem technologisch-orientierten Zugang, d. h. mit der dominanten Logik der Ersten Moderne wird übersehen, dass weder politisch-planerische Strategien noch die Alltagswelt von BürgerInnen im Gegensatz zur mathematischen Logik nicht deterministisch organisiert sind (vgl. Häußling 2014: 218) - es komme vielmehr darauf an, mit welchen Interessen und mit welcher

20 Tarmann (2018: 40) sieht das „autonome Fahren“ in der Phase des „technologischen Transfers“. Diese Einschätzung teile ich nicht, weil zum einen nicht sicher ist, ob nicht weitere Erfindungen notwendig sein werden (Phase 1). Zum anderen ist die ökonomische, ökologische, politische und soziale Einbettung (Phase 2) bislang - mit vereinzelten Ausnahmen in Südostasien und Kalifornien - völlig offen. 
Macht die jeweiligen institutionellen Akteurlnnen innerhalb eines „Handlungskorridors“ agieren (vgl. Mayntz/Scharpf 1995). Die ambivalenten individuellen Handlungen sind daher immer im Kontext widersprüchlicher Systemdynamiken, zeitlicher Flexibilisierungen und räumlicher Fragmentierung zu denken (vgl. Schwedes 2013: 284).

Es reicht also nicht aus, die potentiellen technologischen und technischen Fähigkeiten von Fahrzeugen, Infrastrukturen und deren Vernetzungen zu beschreiben und mittels Szenarien mögliche Auswirkungen auf den Verkehr und die räumliche Entwicklung vorauszusehen, sondern nach Cresswell (2006: 3f.) kommt es vor allem darauf an, wie der avV thematisiert, interpretiert und auf verschiedene Weise als "kommendes und unausweichliches Faktum“ präsentiert wird. In diesem Kontext spielen technische Machbarkeit, politisch-planerische und forschungspolitische Rahmensetzung, Werbung und Marketing zur Mobilität der Zukunft und entsprechende Bilder futuristischer Fahrzeuge und von (Stadt-)Landschaften ${ }^{21}$ sowie (sozial) wissenschaftliche Deutungen über gegenwärtige Gesellschaften und Zukunftstrends für die Ausformung von Ideologien über den avV eine wesentliche Rolle.

Diese analytische Trennung in „objektive Fakten“ und „Konstruktion von Realitäten“ lässt sich im foucaultschen Sinne des „archäologischen Zugangs“ (vgl. Foucault 1972) nicht aufrechterhalten, sondern sollte - die Überlegungen von Frello (2008) auf den avV angewandt - auf die Frage eingehen, wie es dazu kommt, dass die Zukunft der Mobilität (und damit auch die Lösung aller Verkehrsprobleme) ausschließlich durch einen hoch- und vollautomatischen Verkehr determiniert sein wird. Fordern Sheller und Urry (2006: 211) auf, die Diskurse um die Mobilität und die Praktiken der Mobilität zu verfolgen, so weist Cresswell (2010: 14) auf die Bedeutung der Machtkonstellationen hin, aus denen die Diskurse und Handlungen zur Mobilität entstehen und gleichzeitig erzeugt und verfestigt werden. Das bedeutet, dass es vor allem die Aufgabe sozialwissenschaftlicher Forschung ist und künftig verstärkt sein sollte, neben der Beschreibung und kritischen Einordnung der (potentiellen) Folgen des avV sich vor allem dem Entstehungszusammenhang und dem Steuerungspotential des avV zuzuwenden.

\section{LITERATUR}

Albrow, M. 1998. Abschied vom Nationalstaat. Frankfurt am Main: Suhrkamp.

Appadurai, A. 1996. Modernity at Large. Cultural Dimensions of Globalization. Minneapolis, MN: University of Minnesota Press.

Bamberg, S. 2004. „Sozialpsychologische Handlungstheorien in der Mobilitätsforschung: Neue theoretische Entwicklungen und praktische Konsequenzen", in Verkehrsgenese - Entstehung von Verkehr sowie Potenziale und Grenzen der Gestaltung einer nachhaltigen Mobilität, hg. v. H. Dalkmann, M. Lanzendorf und J. Scheiner. Mannheim: MetaGIS Infosysteme, 51-70.

Baumann, Z. 2000. Liquid Modernity. Cambridge: Polity Press.

Beck, S., J. Niewöhner und E. Sørensen 2012. Science and Technology Studies. Eine sozialanthropologische Einführung. Bielefeld: transcript.

Beck, U. 1983. „Jenseits von Klasse und Stand? Soziale Ungleichheiten, gesellschaftliche Individualisierungsprozesse und die Entstehung neuer sozialer Formationen und Identitäten“, in Soziale Ungleichheiten, hg. v. R. Kreckel. Göttingen: Schwartz, 35-74.

21 In den meisten Renderings über künftige urbane Mobilität werden meist lebendige Nutzungen des öffentlichen Raums durch andere Verkehrsträger, in jedem Fall aber Hinweise auf soziale Problematiken oder soziale Ungleichheiten komplett ausgeblendet. 
Beck, U. 1987. Risikogesellschaft. Auf dem Weg in eine andere Moderne. Frankfurt am Main: Suhrkamp.

Beck, U. 1995. „Die ,Individualisierungsdebatte“", in Soziologie in Deutschland. Entwicklung, Institutionalisierung und Berufsfelder, theoretische Kontroversen, hg. v. B. Schäfers. Opladen: Leske + Budrich, 185-198.

Beck, U. 1997. Was ist Globalisierung? Irrtümer des Globalismus - Antworten auf Globalisierung. Frankfurt am Main: Suhrkamp.

Beck, U. 2007. Weltrisikogesellschaft. Auf der Suche nach der verlorenen Sicherheit. Frankfurt am Main: Suhrkamp

Beck, U. 2016. The Metamorphosis of the World. How Climate Change is Transforming Our Concept of the World. Cambridge/Malden, MA: Polity.

Beck, U., W. Bonß und C. Lau 2001. „Theorie reflexiver Modernisierung - Fragestellungen, Hypothesen, Forschungsprogramme“, in Die Modernisierung der Moderne, hg. v. U. Beck und W. Bonß. Frankfurt am Main: Suhrkamp, 11-59.

Beck, U., W. Bonß und C. Lau 2004. „Entgrenzung erzwingt Entscheidung: Was ist neu an der Theorie reflexiver Modernisierung?", in Entgrenzung und Entscheidung, hg. v. U. Beck und C. Lau. Frankfurt am Main: Suhrkamp, 13-61.

Beck, U., A. Giddens und S. Lash 1996. Reflexive Modernisierung. Eine Kontroverse. Frankfurt am Main: Suhrkamp.

Berger, P. L., und T. Luckmann 1969. Die gesellschaftliche Konstruktion der Wirklichkeit. Eine Theorie der Wissenssoziologie. Frankfurt am Main: Fischer.

Berkhout, F., A. Smith und A. Stirling 2004. „Socio-technical regimes and transition contexts“, in System Innovation and the Transition to Sustainability: Theory, Evidence and Policy, hg. v. B. Elzen, F. W. Geels und K. Green. Cheltenham: Edward Elgar, 48-75.

Bijker, W., T. P. Hughes und T. J. Pinch (Hrsg.) 1987. The Social Construction of Technological Systems: New Directions in the Sociology and History of Technology. Cambridge, MA: MIT Press.

Bloor, D. 1999. „The Strong Programme in the Sociology of Science“, in Scientific Inquiry, hg. v. R. Klee. New York: Oxford University Press.

Bonß, W., und S. Kesselring 2001. „Mobilität am Übergang von der Ersten zur Zweiten Moderne“, in Die Modernisierung der Moderne, hg. v. U. Beck und W. Bonß. Frankfurt am Main: Suhrkamp, 177-190.

Braun, S., S. Schatzinger, C. Schaufler, C.-M. Rutka und N. Fanderl 2019. „Autonomes Fahren im Kontext der Stadt von morgen (AFKOS)“. Stuttgart: Fraunhofer IAO. http://publica.fraunhofer.de/eprints/ urn_nbn_de_0011-n-5436689.pdf (23.6.2020).

Brenner, N. 1998. „Between fixity and motion: accumulation, territorial organization and the historical geography of spatial scales“, in Environment and Planning D: Society and Space 16, 459-481.

Brenner, N. 2019. New Urban Spaces: Urban Theory and the Scale Question. Oxford: Oxford University Press.

Callon, M. 1999. „Actor-network theory - the market test“, in Actor Network Theory and After, hg. v. J. Law und J. Hassard. Oxford/Malden, MA: Blackwell, 181-195.

Canzler, W., und A. Knie 2016. „Mobility in the age of digital modernity: Why the private car is losing its significance, intermodal transport is winning and why digitalisation is the key", in Applied Mobilities (1) 1, 56-67.

Canzler, W. und A. Knie 2019. „Autodämmerung: Experimentierräume für die Verkehrswende“, Strategiepapier. Berlin: Heinrich-Böll-Stiftung. DOI: 10.25530/03552.4.

Castells, M. 1989. The Informational City: Information Technology, Economic Restructuring, and the Urban-Regional Process. Oxford/Cambridge: Blackwell.

Castells, M. 2001. Der Aufstieg der Netzwerkgesellschaft, Teil I der Trilogie „Das Informationszeitalter“. Opladen: Leske+Budrich.

Christensen, C. M. 1997. The Innovator's Dilemma: When New Technologies Cause Great Firms to Fail. Boston: Harvard Business School Press.

Collins, H. 1981. „Stages in the Empirical Programme of Relativism“, in Social Studies of Science 11, 3-10.

Cresswell, T. 2006. On the Move. Mobility in the Modern Western World. New York/London: Routledge. 
Cresswell, T. 2010. „Towards a Politics of Mobility“, in Environment and Planning D: Society and Space (28) 1, 17-31.

Cudworth, E., P. Senker und K. Walker 2013. „Introduction: Contested Futures: Technology, Inequality and Progress", in Technology, Society and Inequality. New Horizons of Contested Futures, hg. v. E. Cudworth, P. Senker und K. Walker. New York: Peter Lang, 1-16.

Dangschat, J. S. 1998. „Klassenstrukturen im Nach-Fordismus“, in Alte Ungleichheiten - Neue Spaltungen, Sozialstrukturanalyse 11, hg. v. P. A. Berger und M. Vester. Opladen: Leske + Budrich, 49-88.

Dangschat, J. S. 2013. „Eine raumbezogene Handlungstheorie zur Erklärung und zum Verstehen von Mobilitätsdifferenzen“, in Mobilitäten und Immobilitäten. Menschen - Ideen - Dinge - Kulturen - Kapital, Blaue Reihe - Dortmunder Beiträge zur Raumplanung 142, hg. v. J. Scheiner, H.-H. Blotevogel, S. Frank, C. Holz-Rau und N. Schuster. Essen: Klartext, 91-104.

Dangschat, J. S. 2015. „Gesellschaftliche Vielfalt - Heraus- oder Überforderung der Raumplanung?“, in: Jahrbuch Raumplanung 2015. Department für Raumplanung, Band 3, hg. v. J. S. Dangschat, M. Getzner, M. Haslinger und S. Zech. Wien/Graz: Neuer Wissenschaftlicher Verlag, 15-38.

Dangschat, J. S. 2017a. „Automatisierter Verkehr - was kommt da auf uns zu?“, in Zeitschrift für Politische Wissenschaft 27, 493-507. DOI: 10.1007/s41358-017-0118-8.

Dangschat, J. S. 2017b. „Wie bewegen sich die (Im-)Mobilen? Ein Beitrag zur Weiterentwicklung der Mobilitätsgenese", in Verkehr und Mobilität zwischen Alltagspraxis und Planungstheorie. Ökologische und soziale Perspektiven, hg. v. M. Wilde, M. Gather, C. Neiberger und J. Scheiner. Wiesbaden: Springer, 25-52. DOI: 10.1007/978-3-658-13701-4_3.

Dangschat, J. S. 2019. „Automatisierte und vernetzte Fahrzeuge - Trojanische Pferde der Digitalisierung?", in Infrastruktur und Mobilität in Zeiten des Klimawandels, Jahrbuch Raumplanung Band 6, hg. v. M. Berger, J. Forster, M. Getzner und P. Hirschler. Wien: Neuer Wissenschaftlicher Verlag, $11-28$.

Dangschat, J. S. 2020. „Gesellschaftlicher Wandel, Raumbezug und Mobilität“, in Wechse/wirkungen von Mobilität und Raumentwicklung im Kontext des gesellschaftlichen Wandels, Forschungsberichte der ARL 14, hg. v. U. Reutter, C. Holz-Rau, J. Albrecht und M. Hülz. Hannover: Akademie für Raumentwicklung in der Leibniz-Gemeinschaft, 32-75.

Dangschat, J. S., und A. Stickler 2020. „Kritische Perspektiven auf eine automatisierte und vernetzte Mobilität", in Digitalisierung und Stadtentwicklung, Jahrbuch StadtRegion 2019/2020, hg. v. G. Simon. Im Druck.

Deleuze, G., und F. Guattari 1992. Tausend Plateaus - Kapitalismus und Schizophrenie II. Berlin: Merve.

Deloitte 2019. „2020 Global Automotive Consumer Study: Is consumer interest in advanced automotive technologies on the move? Global focus countries“. https://tinyurl.com/ybrtrlfu (30.4.2019).

Deloitte 2020. „2020 Global Automotive Consumer Study: Is consumer interest in advanced automotive technologies on the move? Europa“. https://tinyurl.com/y87563bo (23.6.2020).

Dolowitz, D. P., und D. Marsh 2000. „Learning from abroad. The role of policy transfer in contemporary policy-making“, in: Governance (13) 1, 5-23.

Dzudzek, I. 2016. Kreativpolitik. Über die Machteffekte einer neuen Regierungsform des Städtischen. Bielefeld: transcript.

Eisenstadt, S. N. 2006. „Die Vielfalt der Moderne: Ein Blick zurück auf die ersten Überlegungen zu den ,Multiple Modernities““, Themenportal Europäische Geschichte. www.europa.clio-online. de/2006/Article=113 (3.4.2020).

Elliott, A. 2018. The Culture of Al. Everyday Life and the Digital Revolution. Milton: Routledge.

Endres, M., K. Manderscheid und C. Mincke 2016. „Discourses and ideologies of mobility: an introduction“, in The Mobilities Paradigm. Discourses and Ideologies, hg. v. M. Endres, K. Manderscheid und C. Mincke. Milton Park: Routledge, 1-7.

Evans, M., und J. Davies 1999. „Understanding policy transfer. A multi-level, multi-disciplinary perspective“, in Public Administration (77) 2, 361-385.

Farias, I., und T. Bender (Hrsg.) 2010. Urban Assemblages: How Actor-Network Theory changes urban studies. London/New York: Routledge.

Florida, R. 2002. The Rise of the Creative Class. And How it's Transforming Work, Leisure, Community and Everyday Life. New York: Basic Books. 
Foerster, H. von 1993. „Mit den Augen des andern“, in Wissen und Gewissen. Versuch einer Brücke, hg. v. S. J. Schmidt. Frankfurt am Main: Suhrkamp, 350-363.

Foucault, M. 1972. Archaeology of Knowledge and the Discourse on Language. New York: Pantheon.

Frello, B. 2008. „Towards a Discursive Analytics of Movement: On the Making and Unmaking of Movement as an Object of Knowledge“, in Mobilities (3) 1, 25-50.

Freudendal-Pedersen, M., S. Kesselring und E. Servou 2019. „What is Smart for the Future City? Mobilities and Automation“, in Sustainability 11, 221. DOI: 10.3390/su11010221.

Friedrichs, J., H. Häußermann und W. Siebel 1986. Süd-Nord-Gefälle in der Bundesrepublik? Sozialwissenschaftliche Analysen. Opladen: Westdeutscher Verlag.

Gad, C., und C. Bruun Jensen 2010. „On the Consequences of Post-ANT“, in Science, Technology \& Human Values (35) 1, 55-80.

Geels, F. W. 2002. „Technological transitions as evolutionary reconfiguration processes: a multi-level perspective and a case-study“, in Research Policy (31) 8/9, 1257-1274.

Geels, F. W. 2004. „From sectoral systems of innovation to socio-technical systems: Insights about dynamics and change from sociology and institutional theory“, in Research Policy (33) 6/7, 897-920.

Geels, F. W. 2005. Technological Transitions and System Innovations: A Co-Evolutionary and SocioTechnical Analysis. Chaltenham: Edward Elgar.

Geels, F. W. 2006. „Multi-Level Perspective on System Innovation: Relevance for Industrial Transformation“, in Understanding Industrial Transformation. Views from Different Disciplines, hg. v. X. Olsthoorn und A. J. Wieczorek. Dordrecht: Springer, 163-186.

Geels, F. W. 2011. „The multi-level perspective on sustainability transitions: Responses to seven criticisms“, in Environmental Innovation and Societal Transitions (1) 1, 24-40. DOI: 10.1016/j. eist.2011.02.002.

Geels, F. W. 2012. „A socio-technical analysis of low-carbon transitions: introducing the multi-level perspective into transport studies“, in Journal of Transport Geography 24, 471-482. DOI: 10.1016/j.jtrangeo.2012.01.021.

Geels, F. W., R. Kemp, G. Dudley und G. Lyons (Hrsg.) 2012. Automobility in Transition? A Socio-Technical Analysis of Sustainable Transport. London: Routledge.

Geels, F. W., und J. W. Schot 2007. „Typology of sociotechnical transition pathways: Refinements and elaborations of the multi-level-perspective“, in Research Policy (36) 3, 399-417.

Genus, A., und A.- M. Coles 2008. „Rethinking the multi-level perspective of technological transitions“, in Research Policy (37) 9, 1436-1445. DOI: 10.1016/j.respol.2008.05.006.

Häußling, R. 2014. Techniksoziologie. Baden-Baden: Nomos.

Hajer, M. A., und H. Wagenaar (Hrsg.) 2003. Deliberative Policy Analysis: Understanding Governance in the Network Society. Cambridge: Cambridge University Press.

Hitzler, R. 1984. „Sinnbasteln: zur subjektiven Aneignung von Lebensstilen“, in Das symbolische Kapital der Lebensstile. Zur Kultursoziologie der Moderne nach Pierre Bourdieu, hg. v. I. Mörth und G. Fröhlich. Frankfurt am Main/New York: Campus, 75-92.

Hoppmann, J. 2015. „The Role of Deployment Policies in Fostering Innovation for Clean Energy Technologies: Insights from the Solar Photovoltaic Industry“, in Business \& Society. A Journal of Interdisciplinary Exploration (54) 4, 540-558.

Hoppmann, J., M. Peters, M. Schneider und V. H. Hoffmann 2013. „The Two Faces of Market Support - How Deployment Policies Affect Technological Exploration and Exploitation in the Solar Photovoltaic Industry“, Research Policy (42) 4, 989-1003. DOI: 10.1016/j.respol.2013.01.002.

Hughes, T. P. 1987. „The Evolution of Large Technological Systems“, in The Social Construction of Technical Systems: New Directions in the Sociology and History of Technology, hg. v. W. E. Bijker, T. P. Hughes und T. Pinch. Cambridge, MA/London: MIT Press, 51-82.

Jing, P., G. Xu, Y. Chen, Y. Shi und F. Zhan 2020. „The Determinants behind the Acceptance of Autonomous Vehicles: A Systematic Review“, in Sustainability 12, 1719. DOI: 10.3390/su12051719.

Jonuschat, H., A. Knie und L. Ruhrort 2016. „Zukunftsfenster in eine disruptive Mobilität. Teil 1: Mobilität in einer vernetzten Welt“. Berlin: Innovationszentrum für Mobilität und gesellschaftlichen Wandel (InnoZ). https://tinyurl.com/yc3zwee3 (22.6.2020). 
Kanger, L., F. W. Geels, B. Sovacool und J. Schot 2018. „Technological diffusion as a process of societal embedding: Lessons from historical automobile transitions for future electric mobility“, in Transportation Research Part D: Transport and Environment 71 (Juni), 47-66.

Kemp, R. 1994. „Technology and the transition to environmental sustainability: the problem of technological regime shifts“, in Futures (26) 10, 1023-1046.

Kemp, R., A. Rip und J. W. Schot 2001. „Constructing transition paths through the management of niches“, in Path Dependence and Creation, hg. v. R. Garud und P. Karnøe. Mahwah, NJ/ London: Lawrence Erlbaum, 269-299.

Kesselring, S. 2008. „The mobile risk society“, in Tracing Mobilities: Towards a Cosmopolitan Perspective, hg. v. W. Canzler, V. Kaufmann und S. Kesselring. Aldershot/Burlington, VT: Ashgate, 77-102.

Kesselring, S. 2020. „Reflexive Mobilitäten“, in Das Risiko - Gedanken über und ins Ungewisse. Interdisziplinäre Aushandlungen des Risikophänomens im Lichte der Reflexiven Moderne. Eine Festschrift für Wolfgang Bonß, hg. v. H. Pelizäus und L. Nieder. Wiesbaden: Springer VS, 155-193.

Knorr-Cetina, K. D. 1989. „Spielarten des Konstruktivismus. Einige Notizen und Anmerkungen“, in Soziale Welt 40 1/2, 86-96.

Künkel, J. 2015. „Urban policy mobilities versus policy transfer“, in sub/urban. zeitschrift für kritische Stadtforschung (3) 1, 7-24.

Läpple, D. 2011. „Das Jahrhundert der Städte und die Diversität städtischer Entwicklungsmuster“, in: Stadt und Urbanität, hg. v. M. Messling, D. Läpple und J. Trabant. Berlin: Kadmos, 34-64.

Lash, S. 1999. Another Modernity: A Different Rationality. Oxford: Blackwell.

Latour, B. 1996. „On actor-network theory“, in Soziale Welt 47, 374.

Latour, B. 2007. Eine neue Soziologie für eine neue Gesellschaft. Einführung in die Akteur-NetzwerkTheorie. Frankfurt am Main: Suhrkamp. Originalausgabe: Reassembling the Social: An Introduction to Actor-Network-Theory, Oxford: Oxford University Press, 2005.

Law, J. 2006. „Notizen zur Akteur-Netzwerk-Theorie: Ordnung, Strategie und Heterogenität“, in $A N$ Thology. Ein einführendes Handbuch zur Akteur-Netzwerk-Theorie, hg. v. A. Bellinger und D. J. Krieger. Bielefeld: transcript.

Mackenzie, D., und L. Wajcman 1985. „Introductory essay: the social shaping of technology“, in The Social Shaping of Technology, hg. v. D. Mackenzie und L. Wajcman. Milton Keynes: Open University Press.

Manderscheid, K. 2012. „Automobilität als raumkonstituierendes Dispositiv der Moderne“, in Die Ordnung der Räume. Geographische Forschung im Anschluss an Michel Foucault, hg. v. H. Füller und B. Michel. Münster: Westfälisches Dampfboot, 145-178.

Manderscheid, K. 2014. „Formierung und Wandel hegemonialer Mobilitätsdispositive: Automobile Subjekte und urban Nomaden“, in Zeitschrift für Diskursforschung (2) 1, 5-31.

Manderscheid, K. 2020. „Antriebs-, Verkehrs- oder Mobilitätswende? Zur Elektrifizierung des Automobilitätsdispositivs“, in Baustelle Elektromobilität. Sozialwissenschaftliche Perspektiven auf die Transformation der (Auto-)Mobilität, hg. v. A. Brunnengräber und T. Haas. Bielefeld: transcript, 37-68.

Marx, Karl 1867/1969. Das Kapital. Kritik der politischen Ökonomie, Band I. Berlin: Dietz.

Mayntz, R., und F. W. Scharpf 1995. „Der Ansatz des akteurzentrierten Institutionalismus“, in Gesellschaftliche Selbstregelung und Steuerung, hg. v. R. Mayntz und F. W. Scharpf. Frankfurt am Main: Campus, 39-72.

McCann, E., und K. Ward 2012. „Policy assemblages, mobilities and mutations. Towards a multi-disciplinary conversation", in Policy Studies (34) 1, 2-18.

McLennan, G. 2004. „Travelling with vehicular ideas. The case of the third way“, in Economy and Society (33) 4, 484-499.

Milakis, D., B. van Arem und B. van Wee 2017. „Policy and society related implications of automated driving: a review of literature and directions for future research“, in Journal of Intelligent Transportation Systems 21 (4), 324-348. DOI: 10.1080/15472450.2017.1291351.

Mincke, C. 2016. „From mobility to its ideology: when mobility becomes an imperative“, in The Mobilities Paradigm. Discourses and Ideologies, hg. v. M. Endres, K. Manderscheid und C. Mincke. Milton Park: Routledge, 11-33. 
Mitteregger, M., E. M. Bruck, A. Soteropoulos, A. Stickler, M. Berger, J. S. Dangschat, R. Scheuvens und I. Banerjee 2020a. AVENUE21. Automatisierter und vernetzter Verkehr: Entwicklungen des urbanen Europa. Berlin: Springer Vieweg.

Mitteregger, M., E. M. Bruck, A. Soteropoulos, A. Stickler, M. Berger, J. S. Dangschat, R. Scheuvens und I. Banerjee 2020b. „Automatisierter und vernetzter Verkehr im Langen Level 4“, in AVENUE21. Automatisierter und vernetzter Verkehr: Entwicklungen des urbanen Europa, hg. v. M. Mitteregger, E. M. Bruck, A. Soteropoulos, A. Stickler, M. Berger, J. S. Dangschat, R. Scheuvens und I. Banerjee. Berlin: Springer Vieweg, 57-99.

Möhrle, M. G. 2018. „Technologie“. https://wirtschaftslexikon.gabler.de/definition/technologie-48435/ version-271688 (24.3.2020).

Nelson, R. R., und S. G. Winter 1982. An Evolutionary Theory of Economic Change. Cambridge, MA: Bellknap.

Peck, J. 2012. „Recreative City. Amsterdam, vehicular ideas, and the adaptive spaces of creative policy“, in International Journal of Urban and Regional Research (36) 3, 462-485.

Peck, J., und N. Theodore 2010. „Mobilizing Policy. Models, methods, and mutations“, in Geoforum (41) 2, 169-174.

Peters, M., M. Schneider, T. Griesshaber und V. H. Hoffmann 2012. „The impact of technology-push and demand-pull policies on technical change - Does the locus of policies matter?", in Research Policy (41) 8, 1296-1308.

Peuker, B. 2010. „Akteur-Netzwerk-Theorie (ANT)“, in Handbuch Netzwerkforschung, hg. v. C. Stegbauer und R. Häußling. Wiesbaden: VS Verlag für Sozialwissenschaften, 325-335.

Pinch, T. J., und W. E. Bijker 1987. „The Social Construction of Facts and Artifacts: Or How the Sociology of Science and the Sociology of Technology Might Benefit Each Other", in The Social Construction of Technical Systems: New Directions in the Sociology and History of Technology, hg. v. W. E. Bijker, T. P. Hughes und T. Pinch. Cambridge, MA/London: MIT Press: 17-50.

Rammert, W. 2003. „Technik in Aktion: Verteiltes Handeln in soziotechnischen Konstellationen“, in Autonome Maschinen, hg. v. T. Christaller und J. Wehner. Wiesbaden: VS Verlag für Sozialwissenschaften, 289-315.

Rammert, W. 2007. Technik-Handeln-Wissen. Zu einer pragmatischen Technik- und Sozialtheorie. Wiesbaden: Springer.

Rip, A., und R. Kemp 1998. „Technological change“, in Human Choice and Climate Change 2, hg. v. S. Rayner und E. L. Malone. Columbus, OH: Battelle, 327-399.

Rosa, H. 2005. Soziale Beschleunigung. Die Veränderung der Zeitstrukturen in der Moderne. Frankfurt am Main: Suhrkamp.

Rupprecht, S., S. Buckley, P. Crist und J. Lappin 2018. „AV-Ready C Cities or ,City-Ready“ AVs?“, in Road Vehicle Automation 4, hg. v. G. Meyer, und S. Beiker. Heidelberg et al.: Springer International Publishing, 223-233.

Saint-Simon, H. de 1814. De la réorganisation de la société européenne. Paris: Delonay.

Santarius, T. 2012. „Der Rebound-Effekt: Über die unerwünschten Folgen der erwünschten Energieeffizienz“, Impulse zur WachstumsWende. Wuppertal: Wuppertal Institut für Klima, Umwelt, Energie.

Sassen, S. 2001. The Global City: New York, London, Tokyo. Princeton, NJ: Princeton University Press.

Scheiner, J. 2009. Sozialer Wandel, Raum und Mobilität. Empirische Untersuchungen zur Subjektivierung der Verkehrsnachfrage. Wiesbaden: VS Verlag für Sozialwissenschaften.

Schmookler, J. 1966. Invention and Economic Growth. Cambridge, MA: Harvard University Press.

Schneidewind, U. et al. 2018. Die Große Transformation. Eine Einführung in die Kunst gesellschaftlichen Wandels. Frankfurt am Main: Fischer.

Schumpeter, J. A. 1942. Capitalism, socialism and democracy. New York/London: Harper.

Schwedes, O. 2013. „Räumliche Mobilität im gesellschaftlichen Transformationsprozess - Ein Resümee“, in Räumliche Mobilität in der Zweiten Moderne. Freiheit und Zwang bei der Standortwahl und Verkehrsverhalten, hg. v. O. Schwedes. Münster: Lit, 273-287.

Sennett, R. 1977. The Fall of Public Man. London/Boston: Faber \& Faber.

Sennett, R. 1998. Der flexible Mensch. Die Kultur des neuen Kapitalismus. München: Siedler. 
Sheller, M. 2011. „Mobility“, in Sociopedia.isa, 1-12; DOI: 10.1177/2056844601163.

Sheller, M., und J. Urry 2006. „The New Mobilities Paradigm“, in Environment and Planning A: Economy and Space (38) 2, 207-226.

Sheller, M., und J. Urry 2016. „Mobilizing the new mobilities paradigm“, in Applied Mobilities (1) 1, 10-25.

Shladover, S. E. 2016. „The Truth about, Self-Driving“ Cars: They are coming, but not the way you may have been led to think", in Scientific American (314) 6, 52-57.

Sovacool, B. K., und J. Axsen 2018. „Functional, symbolic and societal frames for automobility: Implications for sustainability transitions“, in Transportation Research Part A: Policy and Practice 118, 730-746. https://tinyurl.com/yae5rufr (23.6.2020).

Stefano, G. di, A. Gambardella und G. Verona 2012. „Technology Push and Demand Pull Perspectives in Innovation Studies: Current Findings and Future Research Directions“, in Research Policy (41) 8, 1283-1295.

Stickler, A. 2020a. „Automatisiertes und vernetztes Fahren als Zukunftsperspektive für Europa? Eine Diskursanalyse der gegenwärtigen europäischen Politik“, in Baustelle Elektromobilität. Sozialwissenschaftliche Perspektiven auf die Transformation der (Auto-) Mobilität, hg. v. A. Brunnengräber und T. Haas. Bielefeld: transcript, 93-116.

Stickler, A. 2020b. „Automobilität im Umbruch? Gegenwärtige Stabilisierungen oder Transformation der automobilen Hegemonie“, unveröffentlichte Dissertation, TU Wien.

Tarmann, P. 2018. „Autonomes Fahren: Chancen und Risikowahrnehmungen. Eine qualitative Analyse der gesellschaftlichen Akzeptanz selbstfahrender Autos“, unveröffentlichte Masterarbeit, Karl-Franzens-Universität Graz.

Urry, J. 2000. Sociology beyond Societies. Mobilities of the Twenty-First Century. London: Routledge.

Urry, J. 2003. Global Complexity. Cambridge: Polity.

Urry, J. 2004. „The ,System“ of Automobility“, in Theory, Culture \& Society (21) 4/5, 25-39.

Urry, J. 2007. Mobilities. Cambridge: Polity.

Urry, J. 2009. „Mobilities and Social Theory“, in The New Blackwell Companion to Social Theory, hg. v. Bryan S. Turner. Hoboken, NJ: Wiley-Blackwell, 477-495.

Urry, J. 2012. „Social networks, mobile lives and social inequalities“, in Journal of Transport Geography 12, 24-30.

Vester, M., P. von Oertzen, H. Geiling, T. Hermann und D. Müller 2001. Soziale Milieus im gesellschaftlichen Strukturwandel. Zwischen Integration und Ausgrenzung. Frankfurt am Main: Suhrkamp.

Wallerstein, I. 2004. World-System Analysis: An Introduction. Durham: Duke University Press.

Welge, M. K. 1992. Planung: Prozesse - Strategien - Maßnahmen. Wiesbaden: Springer Gabler.

Weyer, J. 2008. Techniksoziologie. Genese, Gestaltung und Steuerung sozio-technischer Systeme. Weinheim/München: Juventa.

Weyer, J. 2019. Die Echtzeitgesellschaft. Wie smarte Technik unser Leben steuert. Frankfurt am Main: Campus.

Wiesner, A. 2003. „Ethnografische Politikforschung“, in Politik als Lernprozess. Wissenszentrierte Ansätze der Politikanalyse, hg. v. M. L. Maier, F. Nullmeier, T. Pritzlaff und A. Wiesner. Wiesbaden: VS Verlag für Sozialwissenschaften, 141-166. 
Open Access Dieser Beitrag wird unter der Creative Commons Namensnennung 4.0 International Lizenz (http://creativecommons.org/licenses/by/4.0/deed.de) veröffentlicht, welche die Nutzung, Vervielfältigung, Bearbeitung, Verbreitung und Wiedergabe in jeglichem Medium und Format erlaubt, sofern Sie den/die ursprünglichen Autor(en) und die Quelle ordnungsgemäß nennen, einen Link zur Creative Commons Lizenz beifügen und angeben, ob Änderungen vorgenommen wurden.

Die in diesem Beitrag enthaltenen Bilder und sonstiges Drittmaterial unterliegen ebenfalls der genannten Creative Commons Lizenz, sofern sich aus der Abbildungslegende nichts anderes ergibt. Sofern das betreffende Material nicht unter der genannten Creative Commons Lizenz steht und die betreffende Handlung nicht nach gesetzlichen Vorschriften erlaubt ist, ist für die oben aufgeführten Weiterverwendungen des Materials die Einwilligung des jeweiligen Rechteinhabers einzuholen.

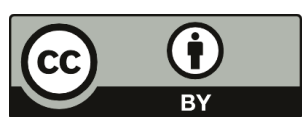

